$\mathrm{PM} / 99-30$

hep-ph/9907360

\title{
Phases in the gaugino sector: direct reconstruction of the basic parameters and impact on the neutralino pair production
}

\author{
J.-L. Kneur 円 and G. Moultaka Z \\ Physique Mathématique et Théorique, UMR No 5825-CNRS, \\ Université Montpellier II, F-34095 Montpellier Cedex 5, France.
}

\begin{abstract}
We consider recovering analytically the (generally complex) parameters $\mu, M_{1}$ and $M_{2}$ of the gaugino and Higgsino Lagrangian, from appropriate physical input in the chargino and neutralino sectors. For given $\tan \beta$, we obtain very simple analytic solutions for $M_{2},|\mu|, \operatorname{Arg}[\mu]$ in the chargino sector and a twofold $\left|M_{1}\right|, \operatorname{Arg}\left[M_{1}\right]$ analytic solution in the neutralino sector, assuming two chargino, two neutralino masses, and one of the chargino mixing angles as physical input. The twofold ambiguity in the neutralino parameters reconstruction may be essentially resolved by measuring the $e^{+} e^{-} \rightarrow \chi_{1}^{0} \chi_{2}^{0}$ production cross-section at future linear collider energies, which we study explicitly with the phase dependences. Some salient features and specific properties of this complex case gaugino "spectrum inversion" are illustrated and compared with the similar inversion in the real case. In particular, our algorithms exhibit in a direct and transparent way the non-trivial theoretical correlation among the chargino and neutralino parameters, and the resulting allowed domains when only a subset of the required physical input masses and production cross-sections is known.
\end{abstract}

\footnotetext{
${ }^{1}$ kneur@lpm.univ-montp2.fr

2 moultaka@lpm.univ-montp2.fr
} 


\section{Introduction}

In the Minimal Supersymmetric extension of the Standard Model (MSSM) [1], 2], without additional theoretical assumptions, the soft-supersymmetry breaking part of the Lagrangian[3] involves a large number of arbitrary parameters. Moreover, many of these parameters may be in general complex, adding new sources of CP violation with respect to the standard model [4, 5]. Apart from the many possible phases in the flavour sector which are mostly severely constrained [6, 7, 5], the other "flavour-blind" possible phases, appearing in particular in the gaugino and/or Higgs sector, are essentially constrained [8] by the electric dipole moment of the electron [9] and the neutron [10. As recently emphasized [11, 12], large phases in this sector are not excluded at present, although it may be considered as unnatural because it generally requires specific cancellations among the different contributing phases 13, 14. Irrespective of a realistic theoretical scenario implying such phases, the latter lead, from a more pragmatic point of view, to potentially drastic changes in the phenomenology of the Higgs and gaugino sectors of the MSSM, therefore affecting the already challenging reconstruction from data [15] - 19] of the structure of the SUSY and soft-SUSY breaking Lagrangian. Typically, assuming that a certain amount of the SUSY partners may be discovered and some of their masses, production cross-sections and decay widths measured, additional questions arise in the presence of non-zero phases. Even without direct measurement of manifestly CP-violating observables, one may for example try to determine whether a set of available data necessarily implies the existence of non-zero phases, or conversely whether the data is consistent with the underlying MSSM basic Lagrangian with phases.

In a previous paper [17], we have given a specific strategy and algorithm to dediagonalize or "invert" the parameters in the ino sector, i.e. obtaining some of the MSSM Lagrangian parameters in direct analytic form in terms of physical parameters (see also ref. 20] for an early such attempt). However, our construction was restricted to the case of real parameters. In the present paper, we generalize our procedure to the complex parameter case. While the techniques used are quite similar, especially for the less straightforward $4 \times 4$ neutralino mass matrix inversion, the choice of the most appropriate input is obviously different, since the number of input/output parameters is necessarily larger when phases are present. In addition, some peculiarities of the complex case require a slightly different approach. As we shall see, it turns out that the complex case inversion is much more constrained than one may think at first: strong correlations between the chargino and neutralino physical masses occur solely from the structure of the MSSM Lagrangian, despite the additional phase freedom, and we will illustrate how this somewhat unconventional spectrum inversion algorithm can exhibit in a more direct and clearer way such non-trivial correlations. For instance, it becomes straightforward in that way to exhibit allowed domains for the neutralino masses, once some of the parameters of the chargino sector are determined (or vice-versa), a task that would usually require rather tedious systematic scanning over the basic Lagrangian parameters with 
painstaking comparisons of physical output results.

For definiteness we shall only consider here the reconstruction of the chargino and neutralino sector parameters with phases, letting aside e.g. the possible implications of non trivial phases on the MSSM Higgs sector phenomenology, which is somehow decoupled from our analysis and has been investigated recently in refs. [21, 22]. Also, as in ref. [17], we emphasize that we are not aiming at a new specific MSSM parameters reconstruction method from experimental observables. Clearly an algebraic approach alone cannot replace more complete studies of the reconstruction of the MSSM parameters from data. Our algorithm should rather be viewed as an efficient tool, to complement any more systematic analysis of the data.

Our brute algorithm gives analytical solutions in the unconstrained MSSM (i.e. no universality of the gaugino mass terms is assumed), for $\mu \equiv|\mu| e^{i \Phi_{\mu}}, M_{2}$, and $M_{1} \equiv\left|M_{1}\right| e^{i \Phi_{M_{1}}}$, using as input two chargino masses $M_{\chi_{1}}, M_{\chi_{2}}$, two (arbitrary) neutralino masses $M_{N_{i}}$, $M_{N_{j}}$, and one chargino mixing angle, plus $\tan \beta$ 円. As will be explained, we can choose a phase convention such that $M_{2}$ is real, so that the counting of physical input versus output parameters is consistent. Obviously, any of the latter input, if not known, may be scanned over some specified range to determine e.g. the resulting allowed domain in the output parameters $\mu, M_{1}, M_{2}$. As a by-product, we obtain the neutralino diagonalization matrix elements - and therefore the neutralino physical couplings - as direct functions of the masses, and we have recalculated the $e^{+} e^{-} \rightarrow \chi_{i}^{0} \chi_{j}^{0}$ production cross-section in this framework with complex $\mu, M_{1}$. The neutralino 1,2 pair production cross-section has been widely studied as a very promising detection process at LEP2 [23, 24, 25] or at a future $e^{+} e^{-}$linear collider [26, 27]. The next- to-lightest $M_{N_{2}}$ mass could be measured with good accuracy, and the lightest (LSP) $M_{N_{1}}$ as well from chargino decay product [26]. As we shall illustrate, the neutralino pair production measurement should also play an important role in resolving an intrinsic ambiguity in the reconstruction of the $M_{1}$ parameter from our algorithm. One should note, however, that prior to our analysis, the precise reconstruction of the chargino masses and mixing angles from actual data will be non trivial. This issue was recently investigated in details in ref. [18, 19, with another way of reconstructing the $\mu$ and $M_{2}$ parameters by considering the chargino pairs which can be produced at a future $e^{+} e^{-}$linear collider and studying theirs spin correlation properties. Accordingly, our analysis is very complementary to theirs, in particular for the neutralino sector.

In section 2, we briefly recall the general parameterization of the gaugino and Higgsino sector, and the physically relevant phases fixing our conventions. In section 3, we discuss the complex chargino and neutralino de-diagonalization algorithm and discuss uniqueness conditions. Typical illustrations of the reconstruction of the parameters are given, and in particular the kind of non-trivial correlations that one can glean in the case of partial knowledge of the input. In section 4 we give analytical expressions for the $e^{+} e^{-} \rightarrow \chi_{i}^{0} \chi_{j}^{0}$

\footnotetext{
${ }^{1}$ This algorithm is available as a fortran code upon request to the authors.
} 
production cross-section in this general complex $\mu, M_{1}$ case and illustrate how it can resolve the two-fold ambiguity for $M_{1}$, when determined from the neutralino masses alone. Finally section 5 gives some conclusions and outlook.

\section{Phases in the MSSM gaugino Lagrangian}

The relevant Gaugino/Higgsino part of the unconstrained MSSM Lagrangian can be found in many different places, see e.g. refs [1, 2]. More specifically the soft SUSY-breaking Majorana mass terms:

$$
\mathcal{L}_{\text {gaugino }}=-\frac{M_{1}}{2} \tilde{B} \tilde{B}-\frac{M_{2}}{2} \tilde{W}^{i} \tilde{W}_{i}-\frac{M_{3}}{2} \tilde{G}^{a} \tilde{G}_{a}+\text { h.c. }
$$

have to be supplemented by the supersymmetric $\mu$ term originating from the quadratic part of the superpotential and contributing to the Higgsino terms. Writing explicitly for illustration only the terms contributing to neutralinos (after electroweak symmetry breaking):

$$
\begin{aligned}
\mathcal{L}_{\text {neutralino }}= & m_{Z} c_{w} \sin \beta \tilde{W}_{3} \tilde{H}_{u}-m_{Z} c_{w} \cos \beta \tilde{W}_{3} \tilde{H}_{d} \\
& +m_{Z} s_{w} \cos \beta \tilde{B} \tilde{H}_{d}-m_{Z} s_{w} \sin \beta \tilde{B} \tilde{H}_{u}+\mu \tilde{H}_{u} \tilde{H}_{d}+\text { h.c. }
\end{aligned}
$$

with $s_{w} \equiv \sin \theta_{W}, c_{w} \equiv \cos \theta_{W}$, and $\tan \beta \equiv v_{u} / v_{d}$ the ratio of the two Higgs vacuum expectation values, $v_{u, d}=\left\langle H_{u, d}\right\rangle$, it is now easy to see from Eqs. 2.1, 2.2) that only the relative phases of say $\left(M_{2}, M_{1}\right)$ and $\left(M_{2}, \mu\right)$ are physically relevant. Indeed any phase change of $M_{2}$ in Eq.(2.1) can always be absorbed by a phase change of the $\tilde{W}$ field. The latter, however, fixes uniquely the phase change of $\tilde{H}_{d}, \tilde{H}_{u}$ and $\tilde{B}$ in Eq.(2.2) since we assume all vev's and gauge couplings to be real, in such a way that the phases of the combinations $M_{2} / M_{1}$ and $\mu M_{2}^{2}$ remain unchanged. We can thus choose, without loss of generality, $M_{2} \geq 0$ and real, and $M_{1}$ and $\mu$ to have arbitrary phases, which fixes our phase convention. Even though no symmetry arguments were invoked here for $\mathcal{L}_{\text {neutralino, }}$ one should keep in mind that Peccei-Quinn and $\mathrm{R}$-symmetries are implicitly needed to assure that phase rotations of the fields, necessary for a given phase convention, preserve automatically the reality of quantities appearing in the PQ- and R-symmetry preserving sector of the MSSM, such as quark and lepton masses and gauge couplings.2]

\section{Extracting the parameters $\mu, M_{2}$ and $M_{1}$}

\footnotetext{
${ }^{2}$ Note that the assumption of no phases for the Higgs vev's is the only convention here that affects the phases of the remaining soft susy sectors of the MSSM. For a clear discussion on the relevant MSSM phases from $U(1)$ symmetry arguments see, e.g., refs. [22, 14].
} 


\subsection{Chargino sector}

In the chargino sector, and with the phase convention discussed above, the mixing mass matrix reads from Eqs. (2.1, 2.2)

$$
M_{C}=\left(\begin{array}{cc}
M_{2} & \sqrt{2} m_{W} \sin \beta \\
\sqrt{2} m_{W} \cos \beta & |\mu| e^{i \Phi_{\mu}}
\end{array}\right)
$$

The squared chargino masses are obtained as the eigenvalues of the $M_{C}^{\dagger} M_{C}$ matrix:

$$
M_{\chi_{1,2}}^{2}=\frac{1}{2}\left[M_{2}^{2}+|\mu|^{2}+2 m_{W}^{2} \mp \Delta\right]
$$

where $\left(\Delta \equiv M_{\chi_{2}}^{2}-M_{\chi_{1}}^{2}\right)$

$$
\Delta=\sqrt{\left(M_{2}^{2}-|\mu|^{2}\right)^{2}+4 m_{W}^{4} \cos ^{2} 2 \beta+4 m_{W}^{2}\left(M_{2}^{2}+|\mu|^{2}\right)+8 m_{W}^{2} M_{2}|\mu| \sin 2 \beta \cos \Phi_{\mu}} .
$$

Note that the diagonalization of the non-symmetric matrix (3.1) is performed via two unitary matrices, involving mixing angles $\left.\phi_{L, R}\right]^{\text {l }}$

$$
U_{L}^{*} M_{C} U_{R}^{-1}=M_{C, \operatorname{diag}}
$$

with

$$
\begin{aligned}
U_{L} & =\left(\begin{array}{cc}
\cos \phi_{L} & e^{-i \beta_{L}} \sin \phi_{L} \\
-e^{i \beta_{L}} \sin \phi_{L} & \cos \phi_{L}
\end{array}\right) \\
U_{R} & =\left(\begin{array}{cc}
e^{i \gamma_{1}} & 0 \\
0 & e^{i \gamma_{2}}
\end{array}\right)\left(\begin{array}{cc}
\cos \phi_{R} & e^{-i \beta_{R}} \sin \phi_{R} \\
-e^{i \beta_{R}} \sin \phi_{R} & \cos \phi_{R}
\end{array}\right)
\end{aligned}
$$

where the phases $\beta_{L, R}$ and $\gamma_{1,2}$ depend on the parameters $M_{2},|\mu|, \Phi_{\mu}$ (and tan $\beta$ ). Their explicit expressions, that we shall not need in our subsequent analysis, may be found e.g. in refs. [19, 11].

From the relations $U_{L}^{*} M_{C} M_{C}^{\dagger}\left(U_{L}^{*}\right)^{-1}=U_{R} M_{C}^{\dagger} M_{C} U_{R}^{-1}=\operatorname{diag}\left(M_{\chi_{2}}^{2}, M_{\chi_{1}}^{2}\right)$ one obtains

$$
\begin{aligned}
\cos 2 \phi_{L} & =\frac{M_{2}^{2}-|\mu|^{2}-2 m_{W}^{2} \cos 2 \beta}{\Delta} \\
\sin 2 \phi_{L} & =\frac{2 m_{W} \sqrt{M_{2}^{2}+|\mu|^{2}+\left(M_{2}^{2}-|\mu|^{2}\right) \cos 2 \beta+2 M_{2}|\mu| \sin 2 \beta \cos \Phi_{\mu}}}{\Delta}
\end{aligned}
$$

and $\phi_{R}$ obtained by similar expressions in which $\sin \beta \leftrightarrow \cos \beta$.

Accordingly, in the complex case there are two independent parameters among $\phi_{L}, \phi_{R}$

\footnotetext{
${ }^{3}$ For convenience we adopt notations similar to those of ref. [19]. Note, however, an overall difference of signs of $\phi_{L}, \phi_{R}$ angles in eqs. (3.6), which should be due to a different convention for the ordering of the two chargino mass eigenvalues. Our convention is such that $M_{C}^{\dagger} M_{C}=\operatorname{diag}\left(M_{\chi_{2}}^{2}, M_{\chi_{1}}^{2}\right)$ (with $\left.M_{\chi_{2}}^{2}>M_{\chi_{1}}^{2}\right)$.
} 
and $\tan \beta$, in addition to the two physical chargino masses. The mixing angles $\phi_{L}$ and $\phi_{R}$ enter in different combinations in the $Z \chi_{i}^{+} \chi_{j}^{-}$and $e \chi_{i}^{-} \nu_{e}$ couplings, such that, as worked out in details in ref. [18, 19, precise measurements of the $e^{+} e^{-} \rightarrow \chi_{i}^{+} \chi_{j}^{-}$cross-section and final state polarization properties should allow a non-ambiguous reconstruction of the two chargino masses, mixing angles $\phi_{L}$ and $\phi_{R}$, and even $\tan \beta$. We refer to their analysis for further details. For convenience, in our subsequent analysis, we rather choose to have $\tan \beta$ as input, having in mind that $\tan \beta$ maybe also determined from another sector.

Therefore, assuming as input $\tan \beta$, the two chargino masses and one mixing angle (say $\phi_{L}$ for definiteness), one can derive straightforwardly from eqs. (3.6) "inverted" expressions for $|\mu|, \Phi_{\mu}$ and $M_{2}$ directly in terms of physical parameters. These read

$$
|\mu|\left(M_{2}\right)=\left[\frac{1}{2}\left(\Sigma-2 m_{W}^{2}\left(1+(-) \cos 2 \beta-(+) \Delta \cos \left(2 \phi_{L}\right)\right)\right]^{1 / 2}\right.
$$

(where $\Sigma \equiv M_{\chi_{2}}^{2}+M_{\chi_{1}}^{2}$ ) and

$$
\begin{aligned}
& \cos \left(\Phi_{\mu}\right)=1-\frac{M_{\chi_{1}}^{2} M_{\chi_{2}}^{2}-\left(P-m_{W}^{2} \sin 2 \beta\right)^{2}}{2 m_{W}^{2} P \sin 2 \beta} \\
& P \equiv M_{2}|\mu|=\frac{1}{2}\left[\left(\Delta \cos 2 \phi_{L}+2 m_{W}^{2} \cos 2 \beta\right)^{2}-\left(\Sigma-2 m_{W}^{2}\right)^{2}\right]^{1 / 2}
\end{aligned}
$$

Actually, since only $\cos \left(\Phi_{\mu}\right)$ enters the mass formulas (3.2), $\Phi_{\mu}$ is determined by (3.8) up to a twofold ambiguity: $\Phi_{\mu} \leftrightarrow 2 \pi-\Phi_{\mu}$. To resolve it requires a priori the measurement of manifestly CP violating observables 19. Note however that the only effect of this ambiguity on our chargino, neutralino inversion algorithm is accordingly $\Phi_{M_{1}} \leftrightarrow-\Phi_{M_{1}}$, without affecting $|\mu|,\left|M_{1}\right|$ or the $e^{+} e^{-} \rightarrow \chi_{i}^{0} \chi_{j}^{0}$ production cross-section, as will be illustrated in subsequent sections.

Now, what is not immediately transparent from eqs. (3.7, 3.8) is that they already exhibit relatively strong constraints among the physical parameters. Namely, arbitrary values of $M_{\chi_{2}}^{2}, M_{\chi_{1}}^{2}$ and $0<\phi_{L}<\pi / 2$, are not at all guaranteed to be consistent with the obvious constraints $M_{2},|\mu| \geq 0$, and $\left|\cos \Phi_{\mu}\right| \leq 1$. More precisely, one arrives, after some straightforward algebra, to the two consistency constraints

$$
\begin{aligned}
& -\left(\Delta^{2} \cos ^{2}\left(2 \phi_{L}\right)+4 m_{W}^{2} \Delta \cos 2 \beta \cos \left(2 \phi_{L}\right)+4 m_{W}^{2} \Sigma-\Delta^{2}-8 m_{W}^{4} \sin ^{2}(2 \beta)\right)^{2} \\
& \leq 16 m_{W}^{4} \sin ^{2}(2 \beta)\left(\Delta \cos \left(2 \phi_{L}\right)+2 m_{W}^{2}(1+\cos 2 \beta)-\Sigma\right) \\
& \times\left(\Delta \cos \left(2 \phi_{L}\right)-2 m_{W}^{2}(1-\cos 2 \beta)+\Sigma\right) \leq 0 \\
& \Delta \cos \left(2 \phi_{L}\right)+2 m_{W}^{2}(1+\cos 2 \beta)-\Sigma \leq 0
\end{aligned}
$$


In other words, only those values of $M_{\chi_{1}}, M_{\chi_{2}}$ and $\cos 2 \phi_{L}$ which satisfy eqs. (3.9) are consistent within the MSSM, which for some masses may give rather restricted ranges for $\cos 2 \phi_{L}$. These algebraic constraints mean that, given $|\mu|$ and $M_{2}$, one cannot reach arbitrary values for the chargino masses despite the phase freedom, the magnitudes of these masses being essentially determined by $|\mu|$ and $M_{2}$. Obviously, the actual chargino pair production will correspond to only one consistent $\phi_{L}$ value, but (3.9) also gives in a straightforward way some other useful information, like for instance the allowed $\left(M_{\chi_{2}}, \phi_{L}\right)$ domain if only the lightest chargino mass is known. Illustrations of such purely theoretical consistency constraints will be given in the next sections.

\subsection{Neutralino sector}

Let us now turn to the de-diagonalization of the neutralino sector. The question we want to answer analytically here, is how to determine in general $\left|M_{1}\right|$ and $\Phi_{M_{1}}$ (as well as two remaining neutralino masses), for given $M_{2},|\mu|, \Phi_{\mu}$, $\tan \beta$ and two arbitrary input neutralino masses $M_{N_{1}}, M_{N_{2}}$ f. At this stage $\mu$ and $M_{2}$ may be either obtained from the previous procedure, eqs. (3.7 3.8), or known by any other mean. Clearly, once the latter $M_{1}$ reconstruction algorithm is obtained, it can easily be combined with the one of the previous section to determine $M_{1}, M_{2}$ and $\mu$ directly from input values of the physical parameters $M_{\chi_{1}}, M_{\chi_{2}}, \phi_{L}, M_{N_{1}}$ and $M_{N_{2}}$, as will be illustrated in section 3.4.

The neutralino mass matrix with the relevant phases reads

$$
M=\left(\begin{array}{cccc}
\left|M_{1}\right| e^{i \Phi_{M_{1}}} & 0 & -m_{Z} s_{W} \cos \beta & m_{Z} s_{W} \sin \beta \\
0 & M_{2} & m_{Z} c_{W} \cos \beta & -m_{Z} c_{W} \sin \beta \\
-m_{Z} s_{W} \cos \beta & m_{Z} c_{W} \cos \beta & 0 & -|\mu| e^{i \Phi_{\mu}} \\
m_{Z} s_{W} \sin \beta & -m_{Z} c_{W} \sin \beta & -|\mu| e^{i \Phi_{\mu}} & 0
\end{array}\right)
$$

Since $M$ is now complex and symmetric but not hermitian, it cannot generally be diagonalized through a similarity transformation (see eq.(4.1)). In order to proceed as in ref[17], we should rather consider the hermitian matrix $M^{\dagger} M$, and use the following four invariants under a similarity transformation:

$$
\begin{aligned}
& T \equiv \operatorname{Tr} M^{\dagger} M=\sum_{i=1,4} M_{N_{i}}^{2} ; \\
& \frac{\left(\operatorname{Tr} M^{\dagger} M\right)^{2}}{2}-\frac{\operatorname{Tr}\left(\left(M^{\dagger} M\right)^{2}\right)}{2}=\sum_{i \neq j} M_{N_{i}}^{2} M_{N_{j}}^{2} ; \\
& \frac{\left(\operatorname{Tr} M^{\dagger} M\right)^{3}}{6}-\frac{\operatorname{Tr} M^{\dagger} M \operatorname{Tr}\left(\left(M^{\dagger} M\right)^{2}\right)}{2}+\frac{\operatorname{Tr}\left(\left(M^{\dagger} M\right)^{3}\right)}{3}=\sum_{i \neq j \neq k} M_{N_{i}}^{2} M_{N_{j}}^{2} M_{N_{k}}^{2} ; \\
& D \equiv \operatorname{Det}^{\dagger} M=\prod_{i=1,4} M_{N_{i}}^{2} ;
\end{aligned}
$$

\footnotetext{
${ }^{4}$ In what follows we denote the two input neutralino masses by $M_{N_{1}}, M_{N_{2}}$ for definiteness and assume them to be the lightest and next to lightest. It should be clear, however, that any two masses among the four will can be equivalently used as input.
} 
where now the $M_{N_{i}}^{2}$ are the (squared) mass eigenvalues satisfying the eigenvalue equation

$$
\operatorname{det}\left(M^{\dagger} M-M_{N}^{2} I\right)
$$

with coefficients of $\left(M_{N}^{2}\right)^{i}$ given by (3.11) for $i=1, . .4$.

After some lengthy but straightforward algebra, we obtain the explicit expressions for (3.11) as a function of the various relevant parameters. Following then a procedure similar to the one discussed in the appendix of ref. [17], but with the obvious replacements $M \rightarrow M^{\dagger} M$ and $M_{N_{i}} \rightarrow M_{N_{i}}^{2}$, we can solve this over constrained system analytically to give $\left|M_{1}\right|, \Phi_{M_{1}}$ and the two remaining physical neutralino masses. It is convenient in a first stage to give the form of the equations controlling $\operatorname{Re}\left[M_{1}\right], \operatorname{Im}\left[M_{1}\right]$ :

$$
\begin{aligned}
& a_{1} \operatorname{Re}\left[M_{1}\right]+b_{1} \operatorname{Im}\left[M_{1}\right]+c_{1}\left|M_{1}\right|^{2}=d_{1} \\
& a_{2} \operatorname{Re}\left[M_{1}\right]+b_{2} \operatorname{Im}\left[M_{1}\right]+c_{2}\left|M_{1}\right|^{2}=d_{2}
\end{aligned}
$$

where the coefficients $a_{i}, b_{i}, c_{i}$ and $d_{i}$ are functions of the parameters $|\mu|, \Phi_{\mu}, M_{2}, M_{N_{1}}$, $M_{N_{2}}$ and $\tan \beta$. Once $\operatorname{Re}\left[M_{1}\right]$ and $\operatorname{Im}\left[M_{1}\right]$ have been determined from (3.13), the remaining unknown (squared) neutralino masses $M_{N_{3}}, M_{N_{4}}$ are obtained as the two solutions of a quadratic equation:

$$
M_{N_{3,4}}^{2}=\frac{1}{2}\left[T-M_{N_{1}}^{2}-M_{N_{2}}^{2} \mp\left(\left(T-M_{N_{1}}^{2}-M_{N_{2}}^{2}\right)^{2}-4 D /\left(M_{N_{1}} M_{N_{2}}\right)^{2}\right)^{1 / 2}\right]
$$

where the trace $T$ and determinant $D$ are given as functions of $\mu, M_{1}, M_{2}$ from eqs.(3.11). The algebraic expressions for $\left|M_{1}\right|, \Phi_{M_{1}}$ are not particularly telling. We thus refrain from giving here more than their generic form explicitly (their fortran encoding does not take, though, more than a couple of dozens of lines).

Rather, the more interesting characteristic features of this neutralino inversion is that, quite similarly to the simpler chargino case discussed above, non-trivial constraints among the physical masses arise, simply because the system (3.13) cannot always have a solution for any arbitrary $M_{N_{1}}, M_{N_{2}}$ input. More precisely, the system is (conditionally) either over constrained and has no consistent solution; or has a twofold solution. This again simply reflects that not all possible $M_{N_{1}}, M_{N_{2}}$ values can be consistently reached when taking into account that $\left|\cos \Phi_{M_{1}}\right|,\left|\sin \Phi_{M_{1}}\right| \leq 1$. (For instance it is clear that the $\Phi_{M_{1}}$ phase freedom cannot compensate for arbitrary values of $|\mu|, M_{2}$ or $\left|M_{1}\right|$, which more essentially determine the magnitude of the two neutralino masses). In the specific form taken by the solution of the system (3.13) this issue is entirely driven by the positivity condition of the radicant $\Delta_{N}$, entering the quadratic solution for $\operatorname{Re}\left[M_{1}\right]$ :

$$
\begin{gathered}
\Delta_{N} \geq 0 \\
\operatorname{Re}\left[M_{1}\right]=\frac{\beta \pm \sqrt{\Delta_{N}}}{2 \alpha}
\end{gathered}
$$




$$
\operatorname{Im}\left[M_{1}\right]=\gamma \operatorname{Re}\left[M_{1}\right]+\delta
$$

where $\Delta_{N}, \alpha, \beta, \gamma$ and $\delta$ have a complicated dependence on $|\mu|, \Phi_{\mu}, M_{2}, M_{N_{1}}, M_{N_{2}}$ and $\tan \beta$, which we do not give here. [The quadratic structure of the solutions is due to the special form of (3.13) where the non-linear terms appear only in $\left|M_{1}\right|^{2}$, otherwise one would have expected a quartic structure.] Accordingly, whenever the system is consistent, i.e. eq. 3.15) satisfied, there are two solutions. It may seem at first sight that this ambiguity occurs only as an artifact of "squaring" the mass matrix in our method. This is not so, for instance even if one considers both $M_{1}$ solutions afterwards in the actual (unsquared) mass matrix (3.10), the ambiguity is not resolved but will be simply reflected in some phases of the mass eigenvalues, or equivalently in the couplings of the corresponding neutralino species. It follows that this twofold ambiguity on $M_{1}$, intrinsic to the case where only two neutralino masses are input, may in fact be resolved by a study of the corresponding neutralino pair production cross-section which we consider in details in section 4 .

\subsection{Reconstruction of $M_{1}$, an illustration}

To put more flesh on the previous algebraic construction, we shall illustrate here some scenarios with typical input choices, and study the consequences on the relevant parameter reconstruction. Let us first consider for simplicity the pure neutralino parameter $\left|M_{1}\right|, \Phi_{M_{1}}$ reconstruction, thus taking $|\mu|, \Phi_{\mu}$ and $M_{2}$ as input. Even though this is not a reconstruction of $M_{1}$ directly from physical parameters, this partial algorithm has some interest before we consider a complete reconstruction from physical chargino and neutralino masses.

In fig. [1 we chose typical input values $|\mu|=100 \mathrm{GeV}, M_{2}=120 \mathrm{GeV}$ (and $\tan \beta=2$ ), such that one of the charginos is relatively light, $M_{\chi_{1}} \simeq 50-120 \mathrm{GeV}$, very roughly within the LEP2 reach or exclusion range . We plot the resulting solutions for $M_{1}\left(\left|M_{1}\right|\right.$ in fig 1a (top) and $\Phi_{M_{1}}$ in fig. 1b (bottom)), as a function of the phase $\Phi_{\mu}$, and for fixed $M_{N_{1}}=40 \mathrm{GeV}$ and $M_{N_{2}}=80 \mathrm{GeV}$ input. (Note that in fig. 1b, the discontinuity in one of the $\Phi_{M_{1}}$ solution is not physical, being only an artifact of the definition of the phase modulo $2 \pi$. Our convention is such that $-\pi<\Phi_{M_{1}}<\pi$ ). The shape of the different plots is rather generic, and several characteristic features can be noticed. First, there is the rather important central zone where no solution for $M_{1}$ exists, corresponding to the consistency constraint (3.15) not being fulfilled. For the input choice in fig. 1, this nosolution zone covers about one third of the possible $\Phi_{\mu}$ values, which is not so restrictive, but it should be noted that a relatively moderate change in the value of either $M_{2} /|\mu|$, $(\tan \beta)$, or $M_{N_{1}} / M_{N_{2}}$ may easily reduce considerably the consistent region, and quite often no consistent $M_{1}$ can be found for any $\Phi_{\mu}$ within a relatively important domain of the

\footnotetext{
${ }^{5}$ One should, however, keep in mind that the existing bounds [7, 25, 28], on top of being model dependent, have been derived assuming real parameters.
} 
input parameter space. This will be illustrated more quantitatively below with "scanning" plots. One can already see the sensitivity of the consistency relation (3.15) to those changes in fig $2 \mathrm{a}, 2 \mathrm{~b}$, where a larger $\tan \beta$ and a lower $M_{N_{2}}$ neutralino mass have been chosen.

In the $\Phi_{\mu}$ range where $M_{1}$ solutions do exist, the plots in fig. 1a,b and 2a,b clearly exhibit the twofold ambiguity on $M_{1}$ (except obviously at the $\Phi_{\mu}$ value(s) such that $\Delta_{N}=0$, the boarder of the no solution zone, where $M_{1}$ is unique and the two distinct curves should meet.) For the input parameter choice in figs. 1 and 2, one observes that the two solutions are much more distinct for $\Phi_{M_{1}}$, than for $\left|M_{1}\right|$ : this is rather generic, although larger differences in the two $\left|M_{1}\right|$ solutions could occur, for instance for very different $M_{N_{1}}, M_{N_{2}}$ values. In fact, as is clear from the figures, the order of magnitude of $\left|M_{1}\right|$ is essentially determined by the values of $|\mu|, M_{2}\left(\right.$ or $\left.M_{\chi_{1}}\right) \simeq \mathcal{O}(100 \mathrm{GeV})$, while the $\Phi_{\mu}$ variation here has a rather moderate influence on $\left|M_{1}\right|$. For example in fig. 1a, $\left|M_{1}\right|$ varies as a function of $\Phi_{\mu}$ roughly between a minimal $\left(\simeq M_{N_{1}}\right)$ and a maximal $\left(\simeq M_{N_{2}}\right)$ value, but that property is somewhat accidentally due to the relatively close values of $|\mu|$ and $M_{2}$ chosen here. When $M_{2} /|\mu|$ is larger (or smaller), we observed that the consistent values of $M_{1}$ are very close to the lightest $M_{N_{1}}$ input values, although it is hard to derive a simple generic behaviour.

\subsection{Complete gaugino inversion, an illustration}

We will now illustrate the merging of the two separate algorithms described in previous sub-sections 3.1 and 3.2 for the chargino and neutralino sectors. The outcome is thus the values of $|\mu|, \Phi_{\mu}, M_{2},\left|M_{1}\right|$ and $\Phi_{M_{1}}$ as direct expressions of the chargino masses and mixing angle $\phi_{L}$, and of the two arbitrary neutralino masses (plus $\tan \beta$ ).

In fig. $3 \mathrm{a}$ and $3 \mathrm{~b}$ are plotted the output modulus and phases respectively, as functions of the physical chargino mixing angle $\phi_{L}$ for typical input chargino and neutralino masses (see figure captions), the lightest being roughly close to present experimental exclusion limits (although again, the exclusion analysis performed assuming all parameters real may not apply here). The non-trivial correlations among the chargino and neutralino masses appear now explicitly. First, and rather generically, the domain where both sectors can consistently exist is quite narrow as a function of $\phi_{L}$ (the right boarder zones in fig. $3 \mathrm{a}, \mathrm{b}$ are first eliminated from inconsistency with the relations (3.9), while the condition $\Delta_{N} \geq 0$ eliminates most of the remaining $\phi_{L}$ domain, leaving the relatively narrow $M_{1}$ solution zones). Again, relatively moderate changes in the input mass values may easily result in narrower or even empty solution zones. For instance, changing $M_{\chi_{1}}$ only from 80 to $100 \mathrm{GeV}$, for the same values of the other input parameters, gives no consistent solutions. Another example is fig. 4 which illustrates the same reconstruction as in fig. 3 but for a slightly higher $\tan \beta$ value. In summary, plots such as the ones in fig. 3 and 4 show that the inversion provides in a direct way the kind of correlations one is expecting among the neutralino masses, once we know the chargino spectrum (or viceversa). We could obviously have illustrated the same kind of information by choosing for 
instance one of the physical masses to vary, instead of $\phi_{L}$ : the plots would be different but would qualitatively reflect the same strong correlations. This will be illustrated more systematically in the next sub-section.

\subsection{Reconstruction from partial input}

We consider now a less optimistic situation where only a partial knowledge of the physical input parameters is assumed, and illustrate the kind of information that can be retrieved in this case. Accordingly in figs. 5-8 some input masses are fixed while other and/or mixing angles are randomly scanned within a reasonable range for the masses (see figure captions). First, in fig.5 we fix the two chargino masses and $\tan \beta$, and vary $\phi_{L}$ between $\phi_{L} \simeq 0.37$ and $\phi_{L} \simeq 0.51(\mathrm{rad})$, which corresponds to the first consistent solution zone in fig. 3a,b. Condition (3.15) implies the pattern of correlation among the physical neutralino masses, represented by the dotted regions ("butterflies") in the figure. More precisely, a given set of the four neutralino masses is consistent only if any pair $\left(M_{N_{i}}, M_{N_{j}}\right)$ of these masses corresponds to a point lying on one of the "butterflies". Definite consequences follow from this requirement such as the fact that each of the three allowed branches (along say the y-axis) can host only one pair $\left(M_{N_{i}}, M_{N_{j}}\right)$. This allows to make qualitative statements about the neutralino spectrum relative to that of the charginos. For instance, with the specific input chosen in fig.5, close inspection shows that there should always be one (and only one) neutralino heavier than the heaviest chargino, and that $M_{\chi_{1}}^{+}-M_{N_{1}}$ gets smaller when $M_{N_{4}}-M_{\chi_{2}}^{+}$gets larger, and vice-versa $\left(M_{N_{1}}\right.$ is the LSP and $M_{N_{4}}$ the heaviest neutralino in our conventions). If $\tan \beta$ increases, the dotted butterflies are simply moving up or down along the diagonal $M_{N_{i}}=M_{N_{j}}$ line, as illustrated for the choice of parameters in fig. 6: thus the (anti)correlation property between the lightest and heaviest mass splitting remains, but for larger $\tan \beta$ values there is room for the four neutralinos being lighter than $M_{\chi_{2}}^{+}$. Finally, note that for the input parameter choice of fig. 5 and 6 , there is no consistent solution when $\tan \beta \gtrsim 8$.

We stress again that these correlations in fig. 5 and 6 are only due to the theoretical consistency relation (3.15), and are simply obtained from scanning over the two input neutralino mass ranges, calculating (3.15) only once for each $M_{N_{i}}, M_{N_{j}}$, (and eventually $\left.\phi_{L}\right)$ input. On top of this, one could easily add any direct experimental constraints on the LSP and other gaugino masses [7, 25, 28] etc (which we however refraine from investigating here, since it is difficult at present to infer which experimental mass limits remain valid when non-zero phases are assumed). From a practical point of view, it may be useful to compare how figs. 5 and 6 could be obtained from a more conventional procedure. Accordingly, one would have to scan over the five-parameter space $M_{2},|\mu|, \Phi_{\mu},\left|M_{1}\right|, \Phi_{M_{1}}$, with $\tan \beta=2(\tan \beta=5)$, diagonalizing the complete chargino and neutralino matrices for each input point, and finally selecting only those output corresponding to the required physical chargino and neutralino masses. Even if our method is not intended to replace conventional procedures of e.g. fitting to the data, it can be in many practical situations an appreciable "theoretical consistency" guide with a substantial gain in simulation time. 
Next, we consider another application of the gaugino spectrum algebraic inversion, where we try to illustrate the kind of minimal data needed to e.g. draw definite conclusions about non-zero phases. Figs. 7 and 8 give typical "contour" plots in the $\left(|\mu|, \Phi_{\mu}\right)$ slice of the basic parameter space, when assuming that only the lightest chargino and the two neutralino masses are fixed, while the heavy chargino mass is basically unknown, and also $\tan \beta$ varies within some range. Figs. 7 and 8 illustrate two different choices for the chargino and the two neutralino masses in this scenario (see figure caption). For the input chosen here, one can see that the "data" definitely imply large non-zero $\Phi_{\mu}$, and this statement does not depend on the specific value of $M_{\chi_{1}}$, as shown by comparing fig. 7 and 8 input. However, when increasing $\tan \beta$ or the splitting between the two neutralino masses, more and more points appear with $\Phi_{\mu}$ close to zero. Qualitatively, one can summarize this behaviour by noting that there exists a well-defined correlated region in the chargino-neutralino mass parameters, such that for small enough $\tan \beta, \Phi_{\mu}$ is necessarily large (the precise value of $\tan \beta$ depending on the precise values of the neutralino mass splitting). It should be thus interesting to simulate this scenario in a more systematic way, taking into account also independent constraints on the phases $\Phi_{\mu}$, $\Phi_{M_{1}}$ [9]-14, on $\tan \beta$, etc, which is however beyond the scope of the present paper.

\section{The $e^{+} e^{-} \rightarrow \chi_{i}^{0} \chi_{j}^{0}$ cross-section with phases}

In this section, we recalculate and illustrate the neutralino pair production in the complex parameter case, in $e^{+} e^{-}$collisions. Our motivation is twofold:

i) once non-zero $M_{1}, \mu$ phases are assumed in the gaugino sector, all predictions for chargino, neutralino production processes will be changed, and it is crucial to analyze the relevant production cross-section with the general phase dependence. This was done for chargino pair production in ref 18, 19] precisely to extract the chargino sector complex parameters. As for the neutralino pair production in $e^{+} e^{-}$collisions, the cross-section with non-trivial phases was calculated in the past 29] and CP-violation effects in the neutralino pair production and decay was also considered in some details [30, 27].

ii) As was stated previously, the reconstruction algorithm, using only two neutralino mass input, leads to a twofold ambiguity in the resulting $M_{1}$ values. We shall illustrate, however, that the above-mentioned $M_{1}$ ambiguity can be in most cases resolved from the corresponding neutralino 1,2 production total cross-section, if the latter is measured with a reasonable accuracy. As a by-product of the inversion procedure, we can also obtain direct correlations between the chargino and neutralino pair production cross-sections. 


\subsection{Analytical expressions}

The neutralino $i, j$ pair production cross-section in $e^{+} e^{-}$collision at tree-level proceeds through s channel $Z, \gamma$ and $\mathrm{t}(\mathrm{u})$-channel sneutrino $\tilde{\nu}_{e}$ exchange. The cross-section for the real parameter case was calculated in ref. [31, 23], and for the complex parameter case in refs [30]. Here we have recalculated this process independently for the full phase dependence in our conventions. In terms of the matrix elements $Z_{i j}$ diagonalizing the neutralino mass matrix (3.10):

$$
Z^{*} M Z^{-1} \equiv M_{\text {diag }}
$$

which are given functions of the parameters $\mu, M_{1}, M_{2}, \tan \beta$, the cross-section reads:

$$
\sigma\left(e^{+} e^{-} \rightarrow \chi_{i}^{0} \chi_{j}^{0}\right)=\frac{\left(2-\delta_{i j}\right)}{2}\left(\sigma_{Z}+\sigma_{\tilde{e}}+\sigma_{Z \tilde{e}}\right)
$$

where the three contributions to the integrated cross-section are given by

$$
\begin{gathered}
\sigma_{Z}=\frac{q}{8 \pi s \sqrt{s}} \frac{g^{4}\left(L_{e}^{2}+R_{e}^{2}\right)}{\cos _{W}^{4}}\left|D_{Z}(s)\right|^{2}\left\{I_{1}\left|O_{j i}^{\prime \prime L}\right|^{2}-2 s m_{i} m_{j} \Re\left[\left(O_{j i}^{\prime \prime L}\right)^{2}\right]\right\} \\
\sigma_{\tilde{e}}=\frac{q}{32 \pi s \sqrt{s}} g^{4}\left\{\left|f_{l_{i}}^{L}\right|^{2}\left|f_{l_{j}}^{L}\right|^{2} I_{3}\left(m_{\tilde{e}_{L}}\right)-s m_{i} m_{j} \Re\left[\left(f_{l_{i}}^{L}\right)^{2}\left(f_{l_{j}}^{L^{*}}\right)^{2}\right] I_{5}\left(m_{\tilde{e}_{L}}\right)+(L \rightarrow R)\right\} \quad(4.4) \\
\sigma_{Z \tilde{e}}=\frac{q}{8 \pi s \sqrt{s}} \frac{g^{4}}{\cos _{W}^{2}} \Re\left[D_{Z}(s)\right]\left\{L_{e}\left(\Re\left[f_{l_{j}}^{L^{*}} f_{l_{i}}^{L} O_{j i}^{\prime \prime}\right] I_{2}\left(m_{\tilde{e}_{L}}\right)-s m_{i} m_{j} \Re\left[f_{l_{j}}^{L^{*}} f_{l_{i}}^{L} O_{j i}^{\prime \prime} L^{*}\right] I_{4}\left(m_{\tilde{e}_{L}}\right)\right)\right. \\
\left.-R_{e}\left(\Re\left[f_{l_{j}}^{R^{*}} f_{l_{i}}^{R} O_{j i}^{\prime \prime} L^{*}\right] I_{2}\left(m_{\tilde{e}_{R}}\right)-s m_{i} m_{j} \Re\left[f_{l_{j}}^{R^{*}} f_{l_{i}}^{R} O_{j i}^{\prime \prime L}\right] I_{4}\left(m_{\tilde{e}_{R}}\right)\right)\right\}
\end{gathered}
$$

where $m_{i}, m_{j}$ designate the two neutralino physical masses, and the various couplings are defined as

$$
\begin{gathered}
f_{l_{i}}^{L}=\sqrt{2}\left[\left(T_{3 l}-e_{l}\right) \tan \theta_{W} Z_{i 1}-T_{3 l} Z_{i 2}\right] \\
f_{l_{i}}^{R}=\sqrt{2} e_{l} \tan \theta_{W} Z_{i 1}^{*} \\
L_{l}=T_{3 l}-e_{l} \sin ^{2} \theta_{W}, \quad R_{l}=-e_{l} \sin ^{2} \theta_{W},
\end{gathered}
$$

and

$$
O_{i j}^{\prime \prime L}=-\frac{1}{2} Z_{i 3} Z_{j 3}^{*}+\frac{1}{2} Z_{i 4} Z_{j 4}^{*}=O_{j i}^{\prime \prime} L^{*}
$$

is the coupling of the $Z$ to neutralino $i, j$ with $O_{i j}^{\prime \prime} R=-O_{i j}^{\prime \prime} L^{*}$ (see the second ref. in [1] for more details, but note a typo in eq.C77 therein.) 
The kinematical variables are

$$
\begin{aligned}
& E_{i}=\sqrt{q^{2}+m_{i}^{2}} \\
& q^{2}=\frac{1}{4 s}\left(s-\left(m_{i}+m_{j}\right)^{2}\right)\left(s-\left(m_{i}-m_{j}\right)^{2}\right) \\
& D_{Z}(s)=\frac{1}{s-m_{Z}^{2}+i m_{Z} \Gamma_{Z}}
\end{aligned}
$$

and the expressions for the integrals $I_{n}$ appearing in eqs. 4.3 4.5) are collected in the appendix, where we also display the relevant expressions for the amplitudes. Note that the complex phase factors in the mass eigenvalues (usually denoted by $\eta_{i}$ [29]-31]) are in our procedure automatically taken into account in eqs. (4.3 4.5), in both CP-conserving and CP-violating cases, through the redefinition

$$
Z_{j k} \rightarrow Z_{j k} e^{i \theta_{j} / 2}
$$

when the mass eigenvalue of the neutralino $j^{\text {th }}$ species picks up a $\theta_{j}$ phase.

Our expressions agree analytically with ref. [31] in the real case and with ref. [29] in the complex case. We obtained as well very good numerical agreement with the values of the cross-section displayed in ref. 23.

\subsection{Illustrations of $\chi_{1}^{0} \chi_{2}^{0}$ production cross-section}

In fig. 9 we plot the $e^{+} e^{-} \rightarrow \chi_{1}^{0} \chi_{2}^{0}$ total cross-section for the same choice of parameters corresponding to fig. 1a,b, and two different choices of the selectron masses (see figure caption), for a LEP2 energy of $190 \mathrm{GeV}$ (top figure) and a future linear collider energy of $500 \mathrm{GeV}$. (For simplicity, we assume a negligible mixing in the selectron sector, so that $m_{\tilde{e}_{L}}$ and $m_{\tilde{e}_{R}}$ are the physical masses). Although our input parameter choice is particular, the behaviour of the cross-section is rather generic. As a first general observation, this plot illustrates the important sensitivity of the total cross-section to the variation of $\Phi_{\mu}$ and $\Phi_{M_{1}}$ (the plots in fig. 9 are functions of $\Phi_{\mu}$ but $\Phi_{M_{1}}$ varies also for this input choice, according to our inversion algorithm, as is clear from the corresponding fig. 1b.) For instance, for both choices of selectron masses illustrated in the figures, the values of the cross-section vary roughly by about $30-40 \%$ when the phases are varied within their maximal possible range, both at LEP2 and linear collider energies. This is not too surprising, since although the two neutralino masses (and the two selectron masses) are fixed, there is an important sensitivity to the phases through the $Z$-neutralinos and $e$ $\tilde{e}$-neutralino couplings entering the cross-section formula, eqs. (4.3 4.5). Now a rather unpleasant feature of the presence of non-zero phases is that, depending on the selectron masses, one may have several different $\Phi_{\mu}\left(\Phi_{M_{1}}\right)$ values giving the same total cross-section value, even though the two neutralino masses and the selectron masses are fixed (see for instance the dotted lines in fig. 9, corresponding to the second $M_{1}$ solution at $190 \mathrm{GeV}$, 
for $m_{\tilde{e}_{L}}, m_{\tilde{e}_{R}}=100,120 \mathrm{GeV}$ ). These ambiguities on the phases may however be easily resolved when looking at the corresponding values of the chargino masses (provided the latter are known), whose corresponding variation with $\Phi_{\mu}$ is illustrated in fig. 1a. This clearly stresses again the importance of looking at possible correlations between the neutralino and chargino sectors in general. Note also that the cross-section values in fig. 9 are symmetric with respect to $\Phi_{\mu} \rightarrow 2 \pi-\Phi_{\mu}$ (which also implies $\Phi_{M_{1}} \rightarrow-\Phi_{M_{1}}$, see e.g. fig. 1b). Concerning now the $M_{1}$ reconstruction ambiguity, which is more specific to our inversion algorithm, the plots in fig. 9 also illustrate that the total cross-section is generally quite sensitive to the twofold $M_{1}$ solution: even though the two $\left|M_{1}\right|$ solutions of fig. 1a are very close to each others, the two solutions for the phases $\Phi_{M_{1}}$ are not, thus leading to the above mentioned sensitivity. Therefore, provided the cross-section is sufficiently large to be measured with a reasonable accuracy [24, 26], one should be able to resolve this ambiguity in our $M_{1}$ reconstruction procedure rather easily, provided of course that the selectron masses are also known.

In fig. 10 we plot the $e^{+} e^{-} \rightarrow \chi_{1}^{0} \chi_{2}^{0}$ total cross-section at LEP2 (top figure) and future linear collider energies (bottom figure), but now as a direct function of the chargino, neutralino masses and chargino mixing angle, for the same choice of parameters corresponding to fig. 3a,b and one choice of the selectron masses (see figure caption). The regions with no plots in fig 10 simply correspond to the excluded $\phi_{L}$ range from consistency of the inversion, as discussed previously for the plots in figs. 3a,b. Again, the different plots illustrate the generically rather important sensitivity of the cross-section to the phases (the plots in fig. 10 are functions of the mixing angle $\phi_{L}$, which corresponds for fixed chargino and neutralino masses to varying $\Phi_{\mu}$ and $\Phi_{M_{1}}$, as is clear from the corresponding fig. $3 \mathrm{~b}$ ). One should keep in mind that since the mixing angle $\phi_{L}$ (more precisely $\cos 2 \phi_{L}$ ) can be in principle determined from the measurement of the chargino pair producion cross-section, plots of the neutralino pair production like in fig.10 may be viewed as a function of the chargino pair production, for fixed chargino and neutralino mass values. This illustrates further the strong correlations between the chargino and neutralino physical parameters. As for the ambiguities in our inversion algorithm, they come in this case too from the twofold solution in the reconstruction of $M_{1}$. Again, given the generically quite different cross-section values corresponding to the two $M_{1}$ solutions in fig. 10, this ambiguity should be easily resolved through the measurement of this observable.

\section{Conclusion}

In the present paper, we have derived a purely algebraic algorithm to reconstruct the (unconstrained) gaugino sector Lagrangian parameters $\mu \equiv|\mu| e^{i \Phi_{\mu}}, M_{2} \equiv\left|M_{2}\right|$ and $M_{1} \equiv\left|M_{1}\right| e^{i \Phi_{M_{1}}}$, directly from the physical chargino and (some of the) neutralino masses. Our construction exhibits in a more direct and systematic way the non trivial correlations among the physical chargino and neutralino physical parameters, which exist even when 
the maximal possible phase freedom of the unconstrained MSSM parameter space is considered, and which may be very hidden or cumbersome to extract in the more standard approach of systematic scanning over the basic parameters. Our approach should be useful in particular in the case where only a subset of the minimal required input is available, and we illustrated with several typical such scenarios the kind of theoretical consistency constraints that could result, in addition to independent experimental constraints.

We have also recalculated the neutralino pair production cross-section $e^{+} e^{-} \rightarrow \chi_{i}^{0} \chi_{j}^{0}$ within our framework and phase conventions, and illustrate its sensitivity to the phases of $\mu$ and $M_{1}$, which should be in particular useful to resolve an ambiguity in the $M_{1}$ parameter reconstruction, intrinsic to our algorithm. A careful comparison of the chargino and neutralino pair production together with the use of the inversion algorithm should also give non trivial correlations among the different chargino/neutralino masses and crosssection observables. Those possible constraints may be studied in a more systematic way, taking into account also the additional direct or indirect constraints from LEP data, measurements of the electric dipole moment, etc...

Finally, a refinement of our tree-level algebraic inversion procedure would be to include radiative corrections. However, these corrections are expected to be generically much smaller for the gaugino masses [32] than for the chargino pair production cross-section [33], and as such, would probably affect more the reconstruction algorithm of the chargino parameters than that of the neutralino ones.

\section{Acknowledgments}

We are thankful to S.Y. Choi, François Richard and Carlos Savoy for useful discussions.

\section{Appendix: Neutralino pair production formulas}

We collect here some formulas needed for the expression of the neutralino $i, j$ pair production cross-section. The integrals in 4.34 .5$)$ read

$$
\begin{aligned}
I_{1} & =\frac{2 s}{3}\left(q^{2}+3 E_{i} E_{j}\right) \\
I_{2}[m] & =\frac{1}{4}\left[\frac{\left(\left(m_{i}^{2}+m_{j}^{2}-2 m^{2}\right)^{2}-s\left(E_{i}-E_{j}\right)^{2}\right)}{q \sqrt{s}} L[m]-4\left(-2 m^{2}+\sqrt{s}\left(E_{i}+E_{j}\right)+m_{i}^{2}+m_{j}^{2}\right)\right] \\
I_{3}[m] & =\frac{1}{2}\left[\frac{8\left(2 s\left(-q^{2}+E_{i} E_{j}\right)-\sqrt{s}\left(E_{i}+E_{j}\right)\left(-2 m^{2}+m_{i}^{2}+m_{j}^{2}\right)+\left(-2 m^{2}+m_{i}^{2}+m_{j}^{2}\right)^{2}\right)}{-4 q^{2} s+\left(-2 m^{2}-\sqrt{s}\left(E_{i}+E_{j}\right)+m_{i}^{2}+m_{j}^{2}\right)^{2}}\right. \\
& \left.+\frac{2 L[m]\left(2 m^{2}-m_{i}^{2}-m_{j}^{2}\right)}{q \sqrt{s}}\right] \\
I_{4}[m] & =\frac{L[m]}{q \sqrt{s}}
\end{aligned}
$$




$$
\begin{aligned}
I_{5}[m] & =\frac{-2 L[m]}{q \sqrt{s}\left(2 m^{2}+\sqrt{s}\left(E_{i}+E_{j}\right)-m_{i}^{2}-m_{j}^{2}\right)} \\
L[m] & =\log \frac{-2 m^{2}+m_{i}^{2}+m_{j}^{2}-\sqrt{s}\left(E_{i}+E_{j}-2 q\right)}{-2 m^{2}+m_{i}^{2}+m_{j}^{2}-\sqrt{s}\left(E_{i}+E_{j}+2 q\right)}
\end{aligned}
$$

The square amplitudes for the neutralino $i, j$ pair production involve $s-, t-$ and $u-$ channel exchanges. They read:

$$
\begin{aligned}
&\left|T_{s}\right|^{2}=4 \frac{g^{4}}{c_{w}^{4}}\left|D_{Z}(s)\right|^{2}\left(L_{e}^{2}+R_{e}^{2}\right)\left\{\left(\xi_{i j}(t)+\xi_{i j}(u)\right)\left|O_{j i}^{\prime \prime L}\right|^{2}-2 s m_{i} m_{j} \Re\left[\left(O_{j i}^{\prime \prime L}\right)^{2}\right]\right\} \\
&\left|T_{t}+T_{u}\right|^{2}= g^{4}\left\{\left|f_{l_{i}}^{L}\right|^{2}\left|f_{l_{j}}^{L}\right|^{2}\left(D_{\tilde{e}_{L}}(t)^{2} \xi_{i j}(t)+D_{\tilde{e}_{L}}(u)^{2} \xi_{i j}(u)\right)\right. \\
&\left.-2 s m_{i} m_{j} D_{\tilde{e}_{L}}(t) D_{\tilde{e}_{L}}(u) \Re\left[\left(f_{l_{i}}^{L}\right)^{2}\left(f_{l_{j}}^{L^{*}}\right)^{2}\right]+(L \rightarrow R)\right\}
\end{aligned}
$$

$$
\begin{aligned}
2 \Re\left[T_{s}\left(T_{t}+T_{u}\right)^{*}\right]=4 \frac{g^{4}}{c_{w}^{2}}\{ \\
L_{e}\left\{D _ { \tilde { e } _ { L } } ( t ) \left[\xi_{i j}(t) \Re\left[D_{Z}(s) f_{l_{j}}^{L} f_{l_{i}}^{L^{*}} O_{j i}^{\prime \prime} L^{*}\right]-s m_{i} m_{j} \Re\left[D_{Z}(s) f_{l_{j}}^{L} f_{l_{i}}^{L^{*}} O_{j i}^{\prime \prime} L_{j}\right]+\right.\right. \\
\left.D_{\tilde{e}_{L}}(u)\left[\xi_{i j}(u) \Re\left[D_{Z}(s) f_{l_{j}}^{L^{*}} f_{l_{i}}^{L} O_{j i}^{\prime \prime}{ }^{\prime}\right]-s m_{i} m_{j} \Re\left[D_{Z}(s) f_{l_{j}}^{L^{*}} f_{l_{i}}^{L} O_{j i}^{\prime \prime} L^{*}\right]\right]\right\} \\
-R_{e}\left\{D_{\tilde{e}_{R}}(t)\left[\xi_{i j}(t) \Re\left[D_{Z}(s) f_{l_{j}}^{R} f_{l_{i}}^{R^{*}} O_{j i}^{\prime \prime}\right]-s m_{i} m_{j} \Re\left[D_{Z}(s) f_{l_{j}}^{R} f_{l_{i}}^{R^{*}} O_{j i}^{\prime \prime} L^{*}\right]\right]+\right. \\
\left.D_{\tilde{e}_{R}}(u)\left[\xi_{i j}(u) \Re\left[D_{Z}(s) f_{l_{j}}^{R^{*}} f_{l_{i}}^{R} O_{j i}^{\prime \prime} L^{*}\right]-s m_{i} m_{j} \Re\left[D_{Z}(s) f_{l_{j}}^{R^{*}} f_{l_{i}}^{R} O_{j i}^{\prime \prime} L\right]\right\}\right\}
\end{aligned}
$$

where

$$
\begin{gathered}
\xi_{i j}(x) \equiv\left(m_{i}^{2}-x\right)\left(m_{j}^{2}-x\right) \\
D_{\tilde{e}_{L, R}}(x)=\frac{1}{x-m_{\tilde{e}_{L, R}}^{2}}
\end{gathered}
$$

and the other functions and couplings as defined in section 4. The differential cross-section is given by

$$
\frac{d \sigma}{d \cos \theta}=\frac{q}{64 \pi s \sqrt{s}}\left(\left|T_{s}\right|^{2}+2 \Re\left[T_{s}\left(T_{t}+T_{u}\right)^{*}\right]+\left|T_{t}+T_{u}\right|^{2}\right)
$$




\section{References}

[1] For reviews see: H.P. Nilles, Phys. Rep. 110, 1 (1984);

H.E. Haber and G.L. Kane, Phys. Rep. 117, 75 (1985);

[2] see for instance S. P. Martin, hep-ph/9709356, in Perspectives in Supersymmetry, ed. by G. L. Kane, World Scientific.

[3] L. Girardello and M.T. Grisaru, Nucl.Phys. B194 (1982) 65.

[4] J. Ellis, S. Ferrara and D. Nanopoulos, Phys. Lett. B114 (1982) 231; W. Buchmüller and D. Wyler, Phys. Lett. B121 (1983) 321; J. Polchinski and M. Wise, Phys. Lett. B125 (1983) 393; F. del Aguila, M. Gavela, J. Grifols and A. Mendez, Phys. Lett. B126 (1983) 71; D. Nanopoulos and M. Srednicki, Phys. Lett. B128 (1983) 61.

[5] For recent reviews on CP violation in supersymmetry and FCNC constraints, see e.g. Y. Grossman, Y. Nir and R. Rattazzi, hep-ph/9701231, in "Heavy flavours II" eds. A. Buras and M. Lindner, World Scientific;

A. Masiero and L. Silvestrini, hep-ph/9711401, Lectures at the Erice school, Sept. 1997.

[6] S. Adler et al, Phys. Rev. Lett. 79 (1997) 2204.

[7] Review of Particle Physics, C. Caso et al., Eur. Phys. J. C 3 (1998) 1.

[8] T. Falk and K.A. Olive, Phys. Lett. B375 (1996) 196; Phys. Lett. B439 (1998) 71.

[9] E. Commins et al, Phys; Rev; A50 (1994) 2960;

K. Abdullah et al, Phys. Rev. Lett. 65 (1990) 234.

[10] P.G. Harris et al, Phys. Rev. Lett. 82, 904 (1999).

[11] T. Ibrahim and P. Nath, Phys. Lett. B418 (1998) 98; Phys. Rev. D57 (1998) 478; Phys. Rev. D58 (1998) 111301.

[12] M. Brhlik and G.L. Kane, Phys. Lett. B437 (1998) 331;

M. Brhlik, G.J. Good and G.L. Kane, Phys. Rev. D59 (1999) 115004; M. Brhlik, L. Everett, G.L. Kane and J. Lykken, hep-ph/9905215.

[13] A. Bartl, T. Gajdosik, W. Porod, P. Stockinger and H. Stremnitzer, hep-ph/9903402.

[14] S. Pokorski, J. Rosiek and C. Savoy, hep-ph/9906206.

[15] I. Hinchliffe et al., Phys. Rev. D55 (1997) 5520; CMS Collaboration (S. Abdullin et al.), hep-ph/9806366;

[16] D. Denegri, W. Majerotto and L. Rurua, Phys. Rev. D58 (1998) 095010. 
[17] J.-L. Kneur and G. Moultaka, Phys. Rev. D59 (1999) 015005.

[18] S.Y. Choi, A. Djouadi, H. Dreiner, J. Kalinowski and P.M. Zerwas, Eur. Phys. J. C7 (1999) 123.

[19] S.Y. Choi, A. Djouadi, H.S. Song and P.M. Zerwas, Eur. Phys. J. C8 (1999) 669; hep-ph/9812236.

[20] A. Bartl et al. Phys. Rev. D40 (1989) 1594.

[21] D. Demir, hep-ph/9901389;

B. Grzadkowski, J.F. Gunion and J. Kalinowski, hep-ph/9902308.

[22] A. Pilaftsis and C.E.M. Wagner, hep-ph/9902371.

[23] S. Ambrosanio and B. Mele, Phys.Rev.D52:3900-3918,1995

[24] Physics at LEP2, CERN 96-01 vol. 1 p.463, eds. G. Altarelli, T. Sjöstrand and F. Zwirner.

[25] ALEPH Collaboration (R. Barate et al.), CERN-EP-99-014, Feb. 1999; DELPHI Collaboration (P. Abreu et al.), Phys. Lett B446 (1999) 75; OPAL Collaboration (G. Abbiendi et al.), Eur. Phys. J. C8 (1999) 255; L3 Collaboration (M. Acciarri et al.), Eur. Phys. J. C4 (1998) 207; CDF collaboration (F. Abe et al.), Phys. Rev. Lett. 80 (1998) 5275.

[26] ECFA/DESY LC Physics Working Group report, Phys. Rept. 299 (1998) 1, and references therein.

[27] G. Moortgat-Pick, H. Fraas, A. Bartl and W. Majerotto, hep-ph/9903220.

[28] for a recent collection of supersymmetric particle LEP limits, see e.g. A. Djouadi, S. Rosier-Lees et al., GDR-SUSY MSSM working group report, hep-ph/9901246.

[29] S.T. Petcov, Phys. Lett. B178 (1986) 57.

[30] N. Oshimo, Z. Phys. C41 (1988) 129; Y. Kizukuri and N. Oshimo, Phys. Lett. B249 (1990) 449.

[31] A. Bartl, H. Fraas and W. Majerotto, Nucl. Phys. B278 (1986) 1

[32] D. Pierce and A. Papadopoulos, Nucl. Phys. B430 (1994) 278.

[33] M.A. Diaz, S.F. King and D. A. Ross, Nucl. Phys. B529 (1998) 23. 

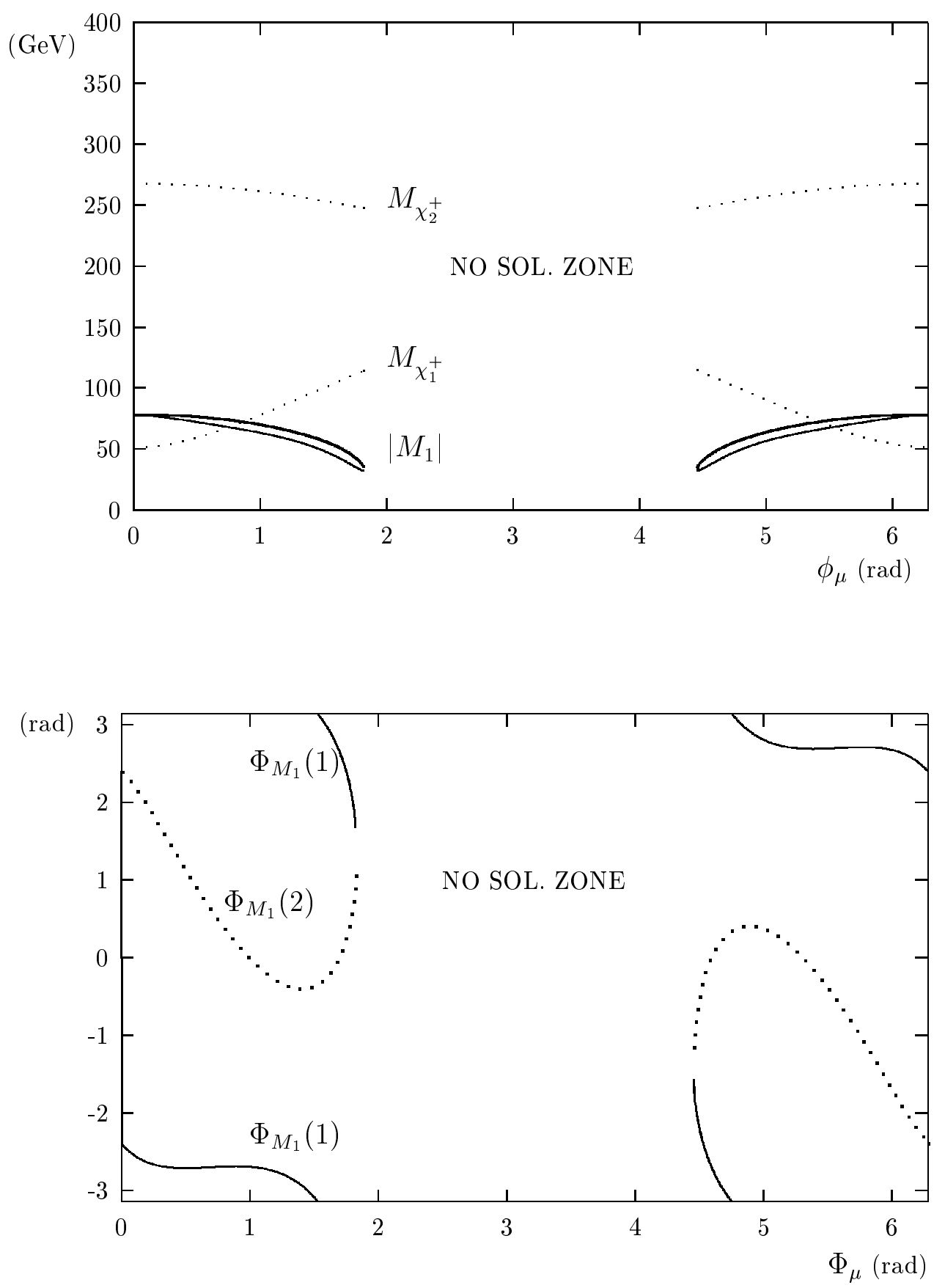

Figure 1: the twofold $\left|M_{1}\right|$ (top figure) and $\Phi_{M_{1}}$ (bottom figure) reconstruction with input choice $|\mu|=100 \mathrm{GeV}, M_{2}=120 \mathrm{GeV}, M_{N_{1}}=40, M_{N_{2}}=80 ; \tan \beta=2$. Also shown are the corresponding chargino mass values. 

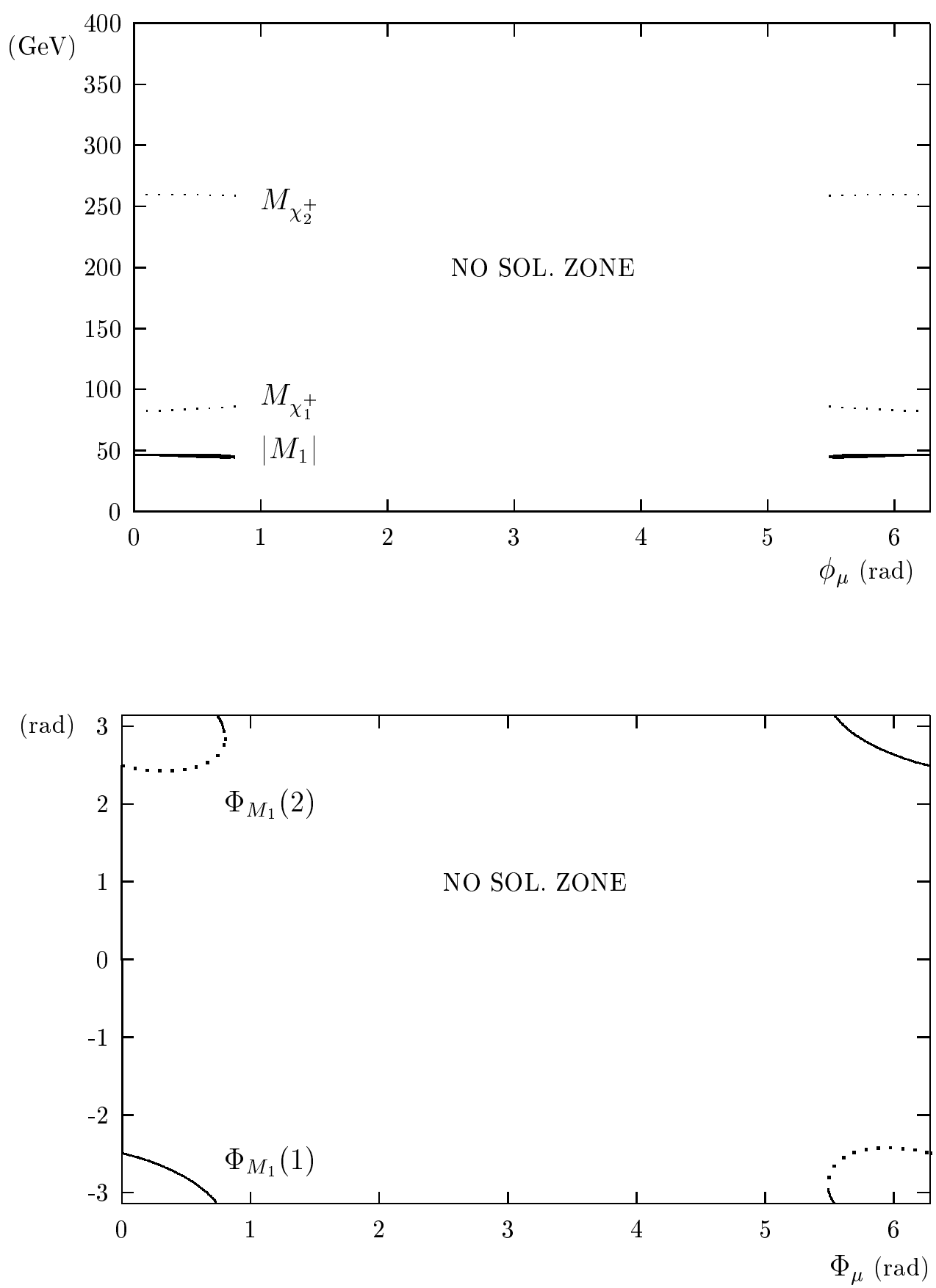

Figure 2: same as fig. 1, with input choice $|\mu|=100 \mathrm{GeV}, M_{2}=120 \mathrm{GeV}, M_{N_{1}}=40$, $M_{N_{2}}=60 ; \tan \beta=10$. 

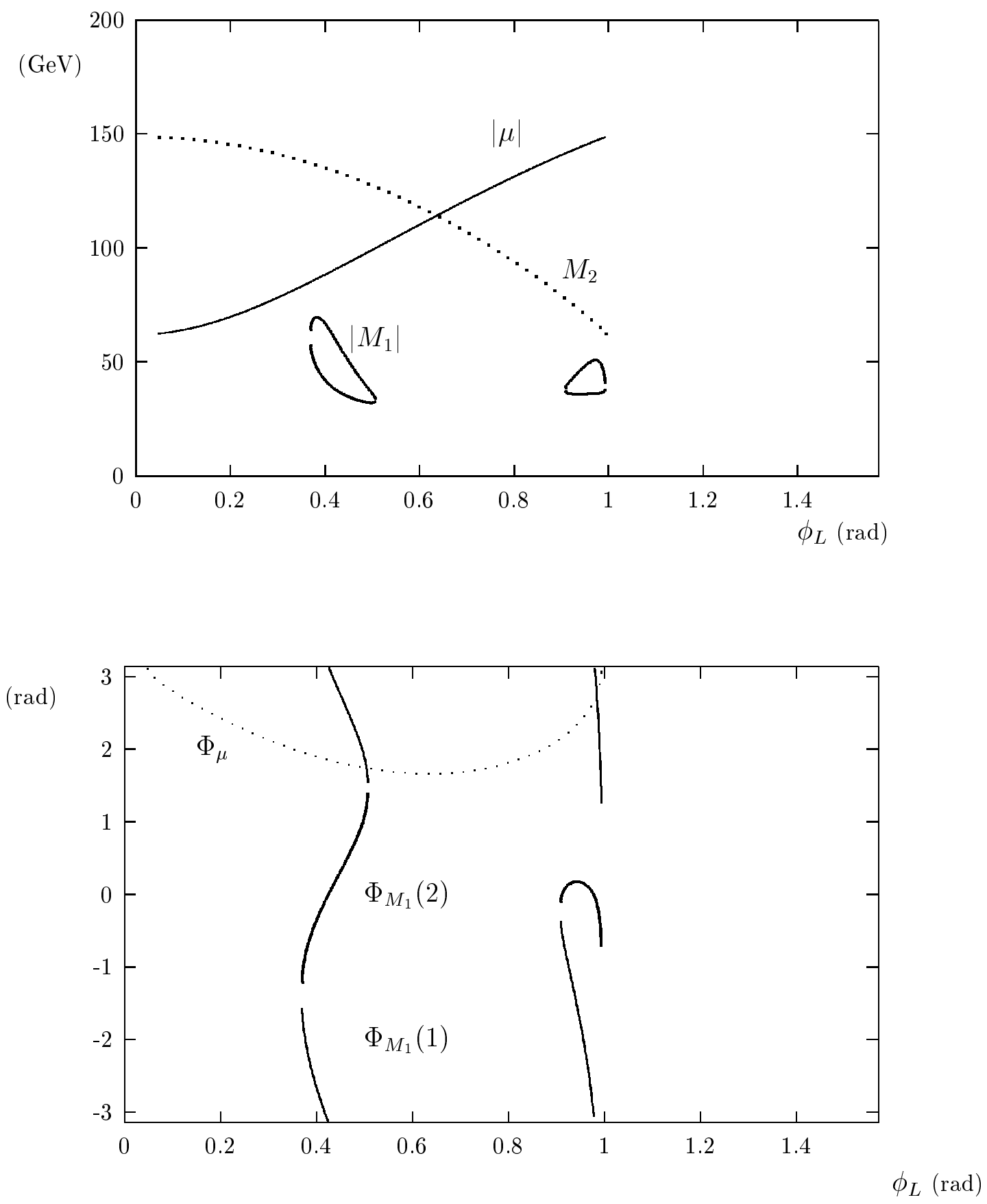

Figure 3: $\left|M_{1}\right|, M_{2},|\mu|$ (top figure) and $\Phi_{\mu}, \Phi_{M_{1}}$ (bottom figure) full reconstruction with physical input choice $M_{\chi_{1}^{+}}=80 \mathrm{GeV}, M_{\chi_{2}^{+}}=180 \mathrm{GeV}, M_{N_{1}}=40, M_{N_{2}}=80 ; \tan \beta=2$. 


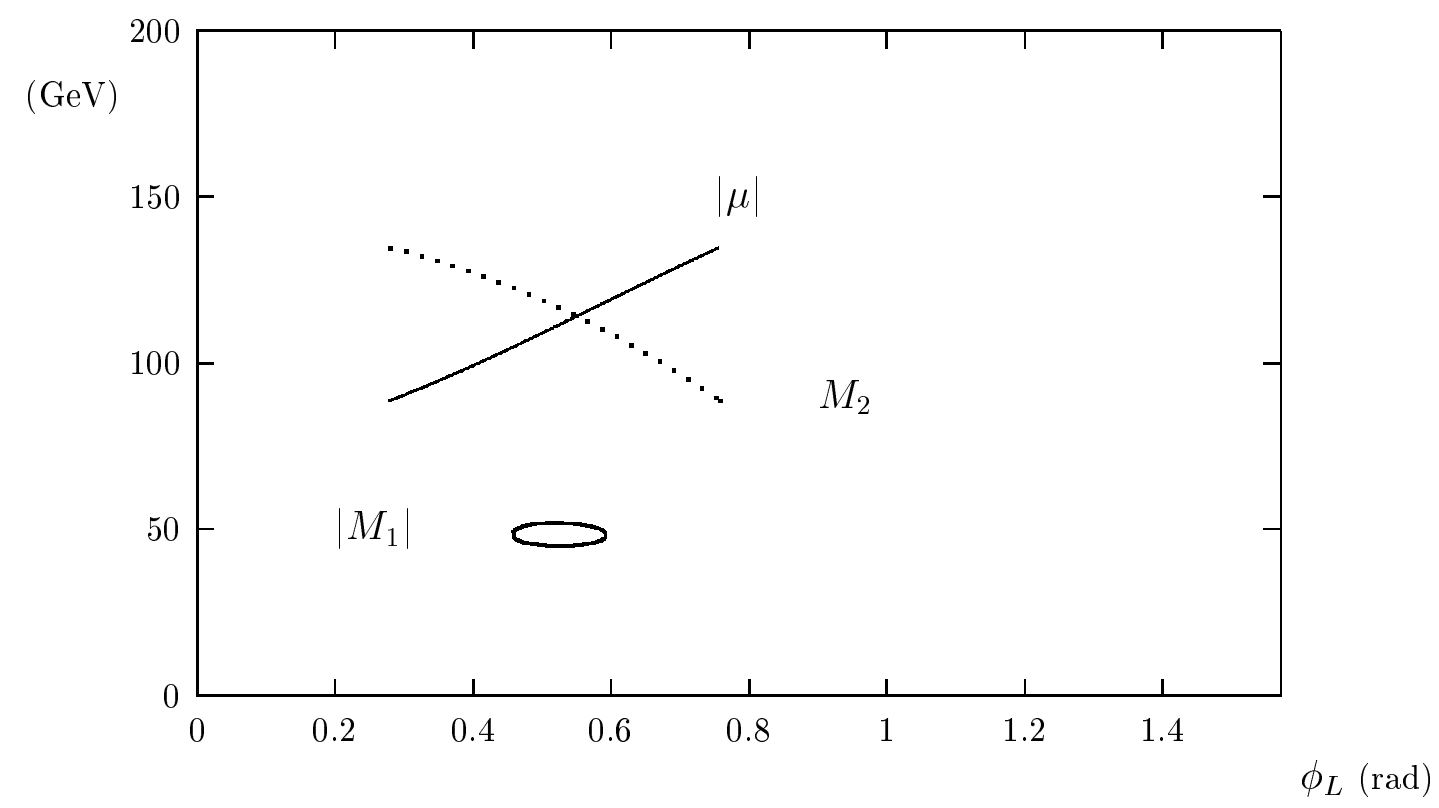

$(\mathrm{rad})$

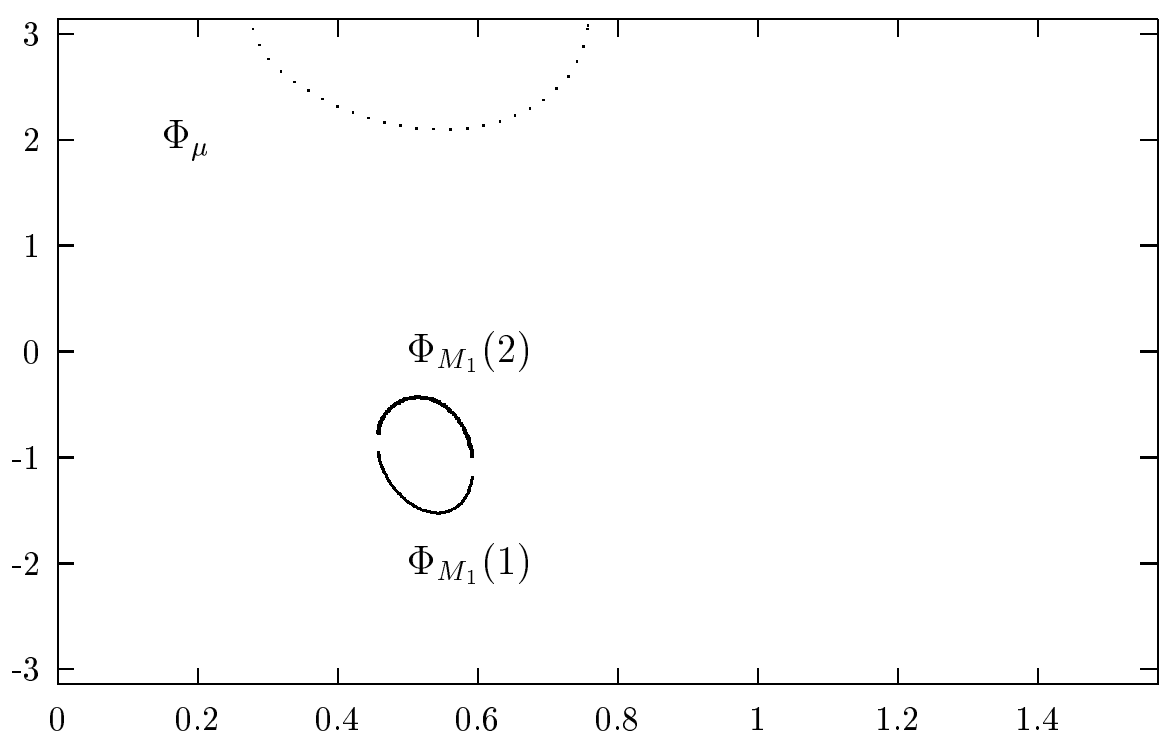

$\phi_{L}(\mathrm{rad})$

Figure 4: same as fig. 3 with input choice $M_{\chi_{1}^{+}}=80 \mathrm{GeV}, M_{\chi_{2}^{+}}=180 \mathrm{GeV}, M_{N_{1}}=40$, $M_{N_{2}}=80 ; \tan \beta=5$. 


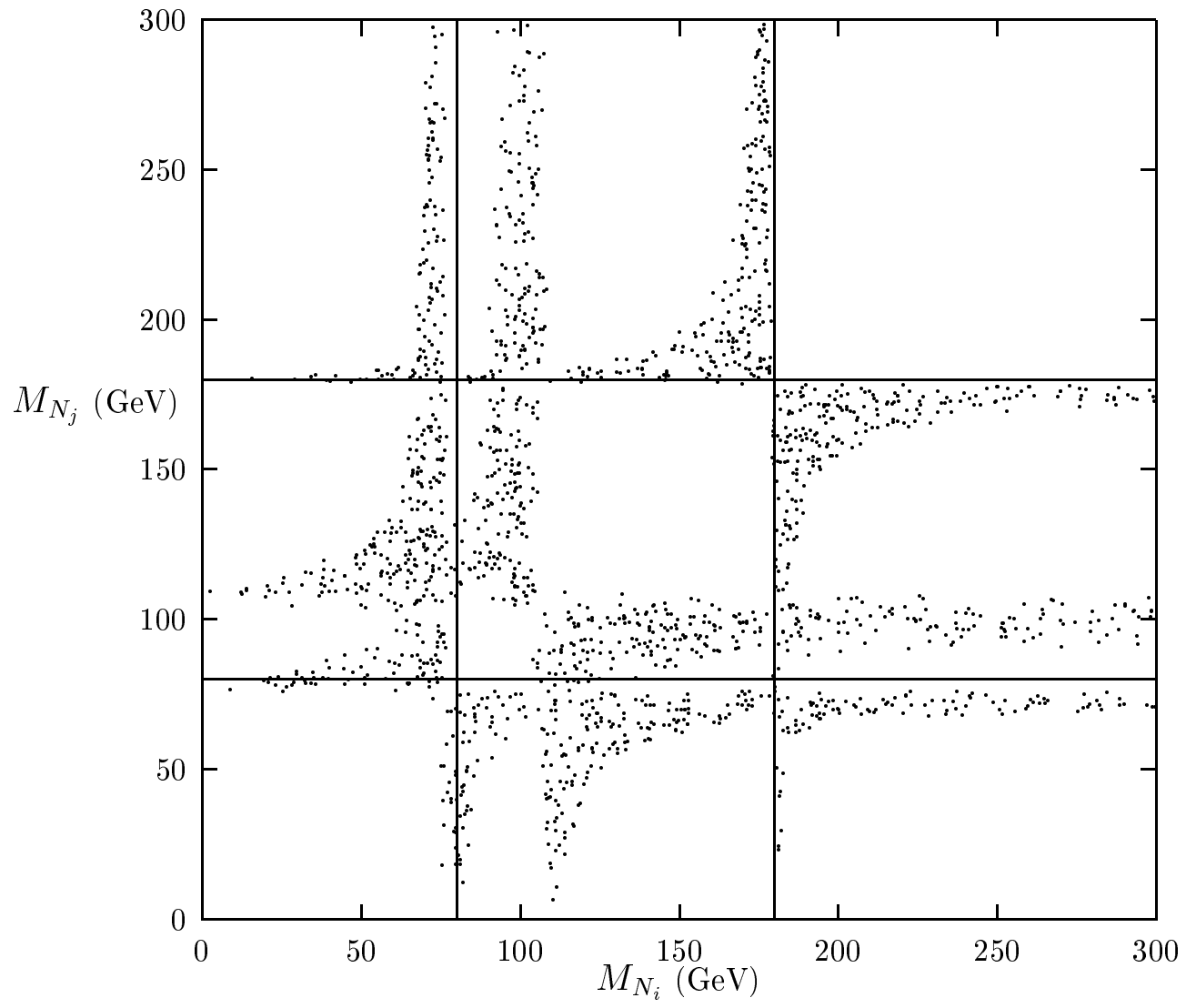

Figure 5: Correlations between arbitrary neutralino masses from consistency of the inversion. Input parameters: $M_{\chi_{1}^{+}}=80 \mathrm{GeV}, M_{\chi_{2}^{+}}=180 \mathrm{GeV}$ (indicated by the two straight lines), $0.37<\phi_{L}(\mathrm{rad})<0.52 ; \tan \beta=2$. 


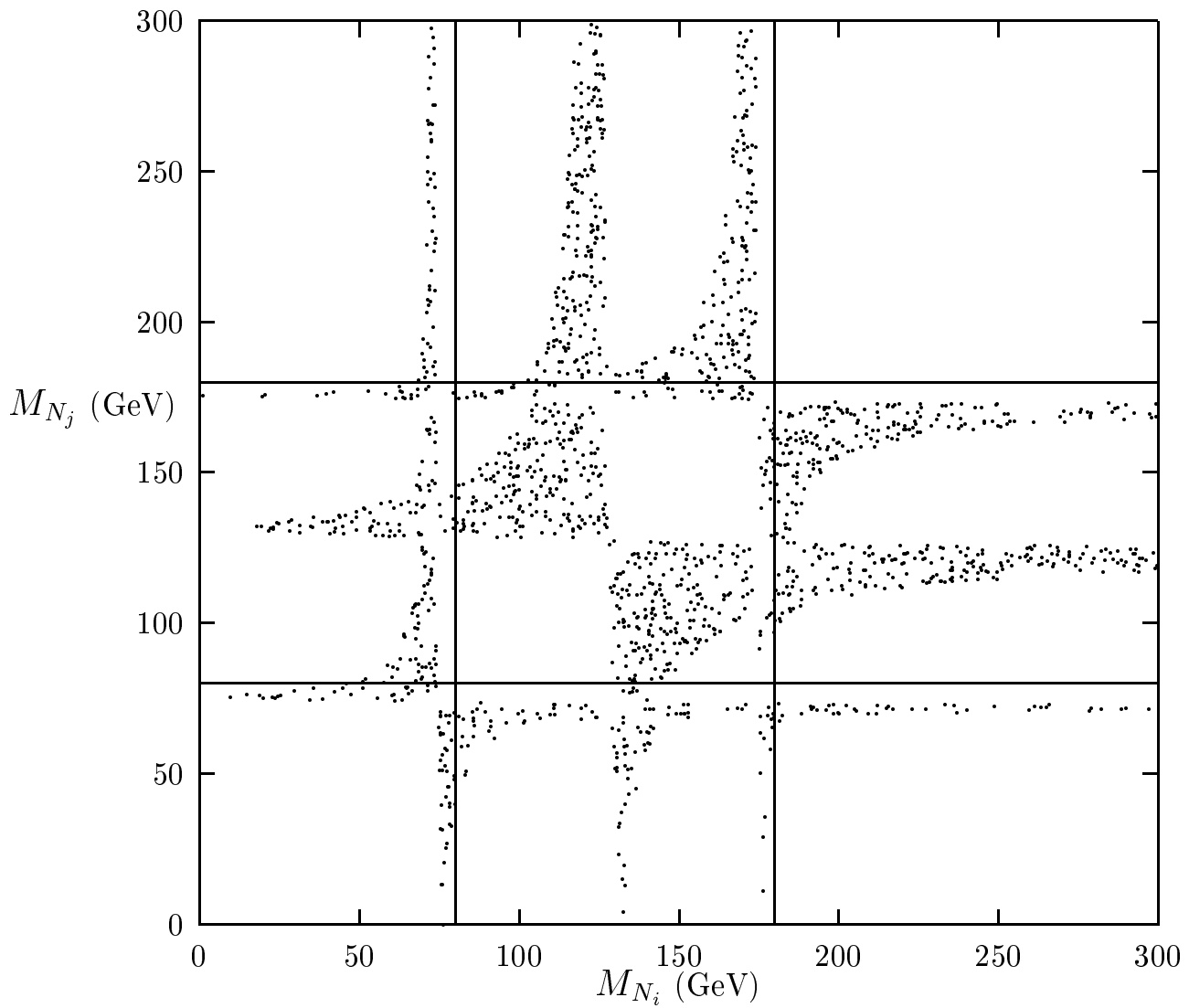

Figure 6: same as fig. 5 for a different input choice: $M_{\chi_{1}^{+}}=80 \mathrm{GeV}, M_{\chi_{2}^{+}}=180 \mathrm{GeV}$, $\phi_{L}(\mathrm{rad})=0.5 ; \tan \beta=5$ 


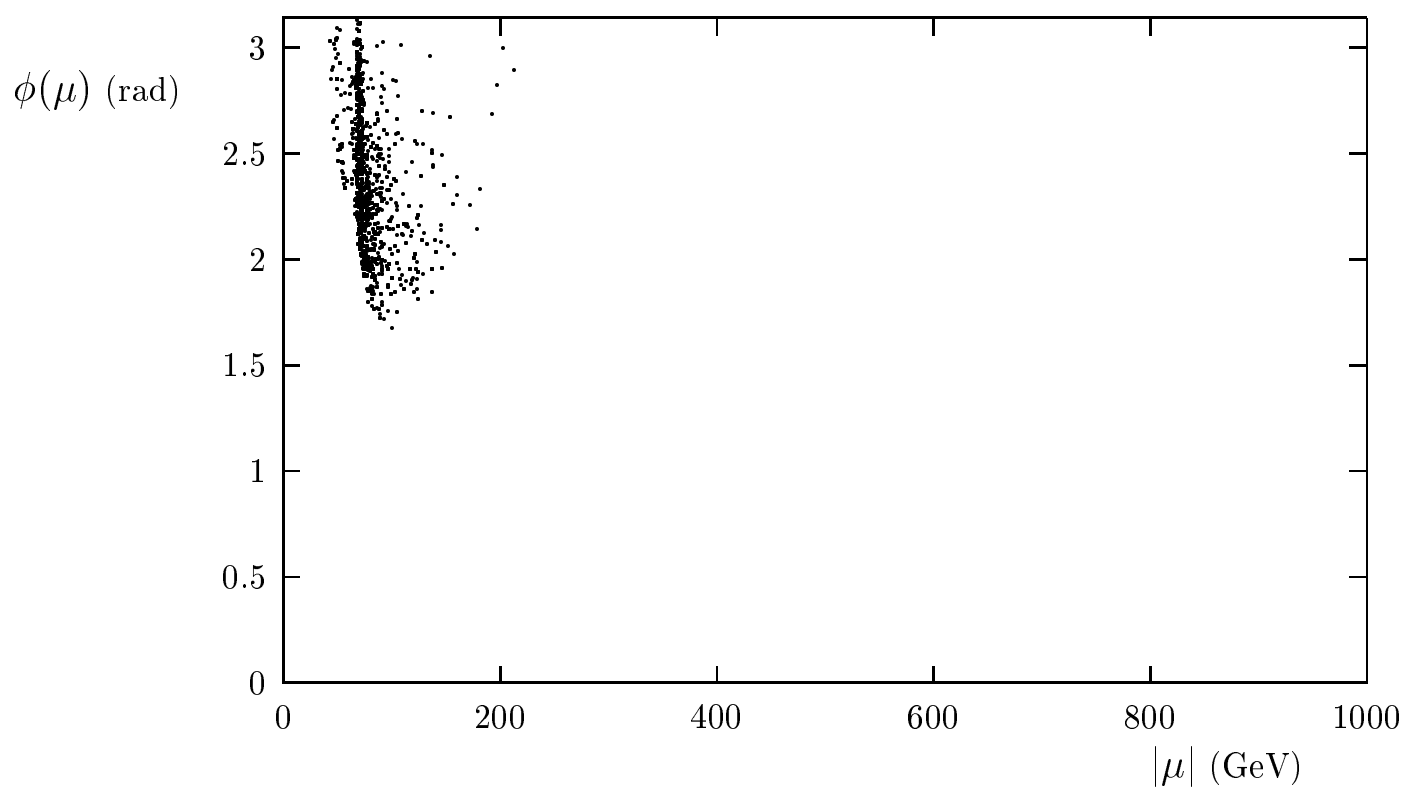

Figure 7: Correlations in the $\left(|\mu|, \Phi_{\mu}\right)$ plane from consistency of the inversion, with incomplete knowledge of the input parameters: $M_{\chi_{1}^{+}}=80 \mathrm{GeV}, 80<M_{\chi_{2}^{+}}<1000$, $0<\phi_{L}<\pi / 2, M_{N_{1}}=40, M_{N_{2}}=70 ; 1<\tan \beta<3$.

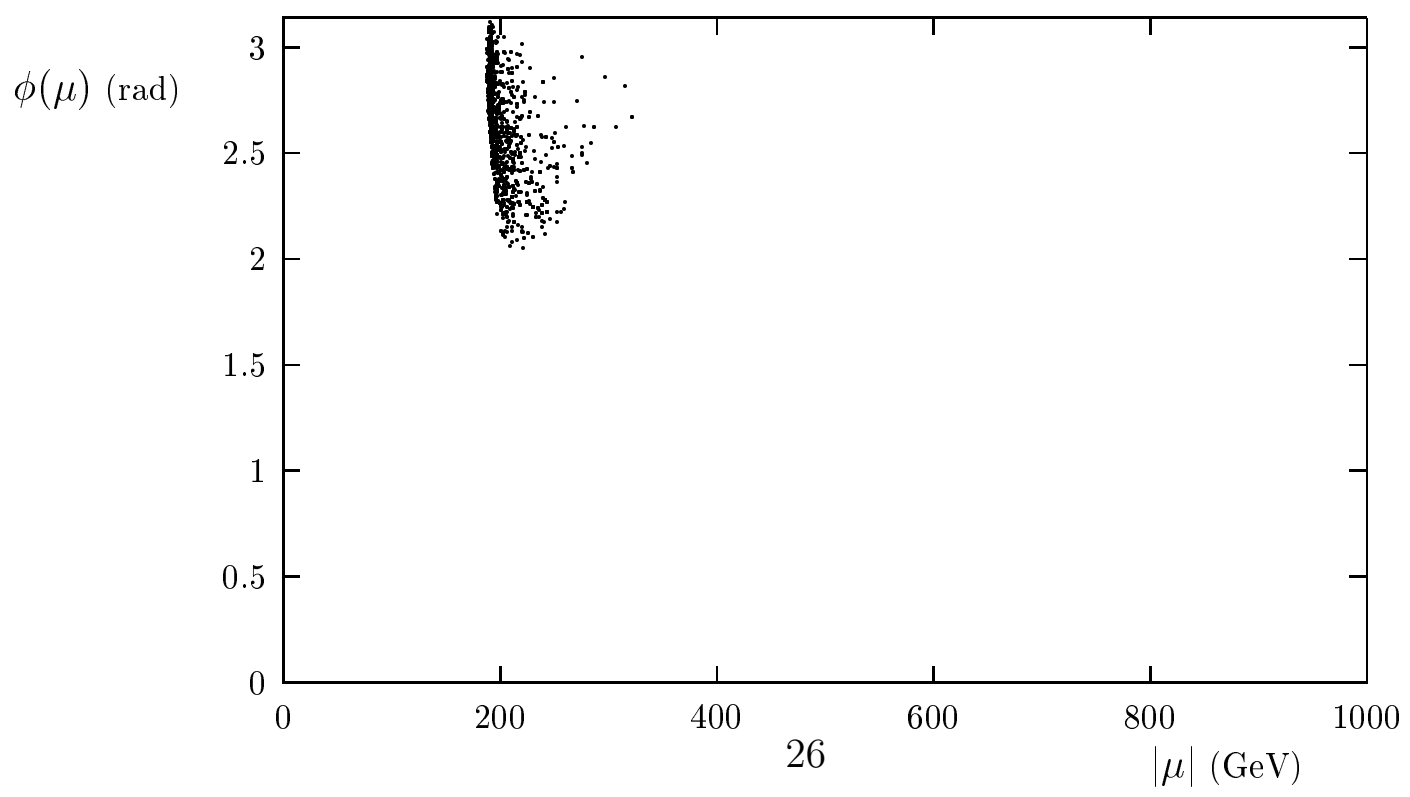

Figure 8: $\quad$ same as fig. 7 with a different input choice: $M_{\chi_{1}^{+}}=200 \mathrm{GeV}, 200<M_{\chi_{2}^{+}}<$ $1000,0<\phi_{L}<\pi / 2, M_{N_{1}}=150, M_{N_{2}}=190 ; 1<\tan \beta<3$. 

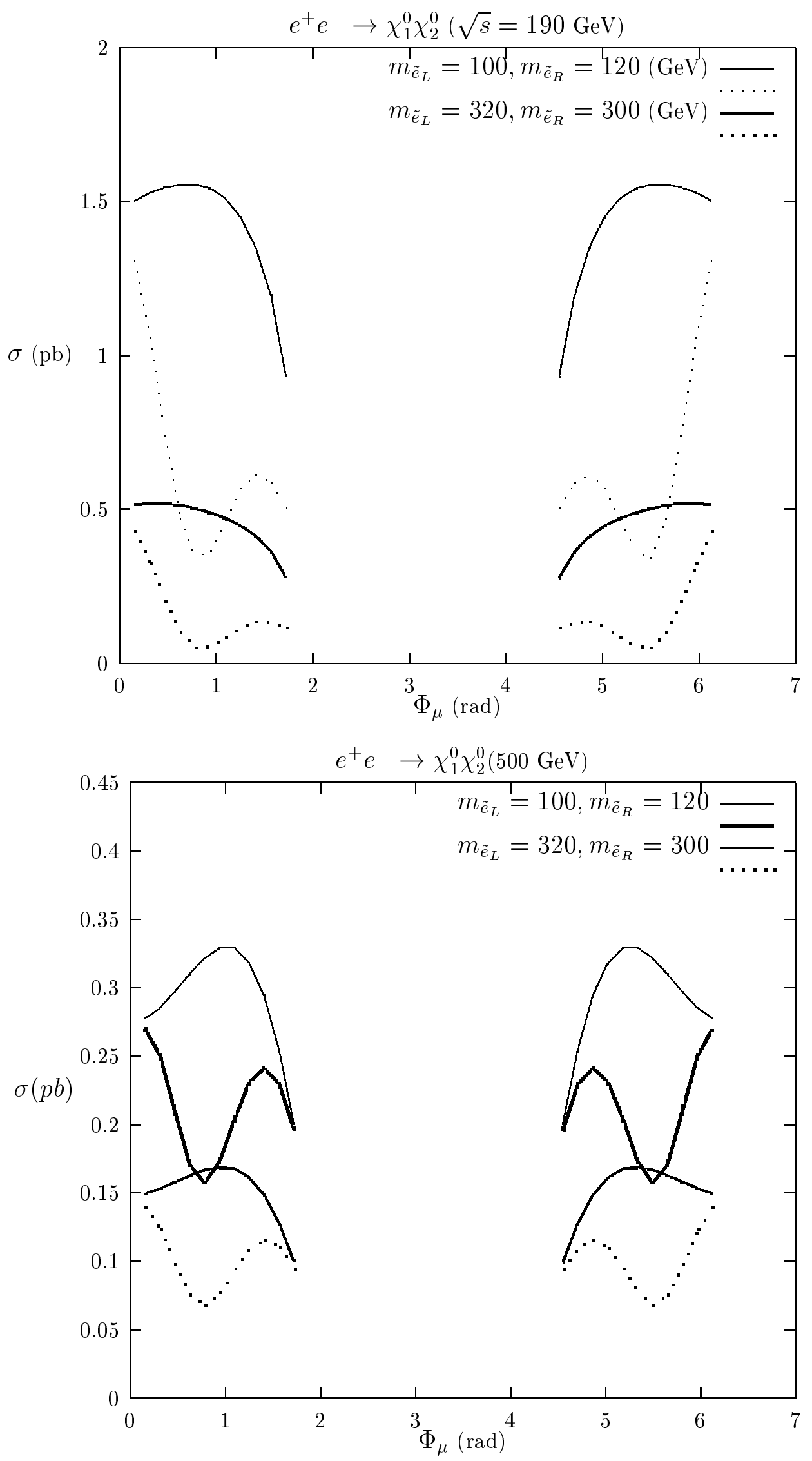

Figure 9: $\quad \chi_{1}^{0}, \chi_{2}^{0}$ production cross-section at $\sqrt{s}=190,500 \mathrm{GeV}$, versus $\Phi_{\mu}$, for two different choices of selectron masses, and input choice as in fig. 1. Plain (dotted) lines correspond to the first (second) $M_{1}$ solution of fig. 1. 

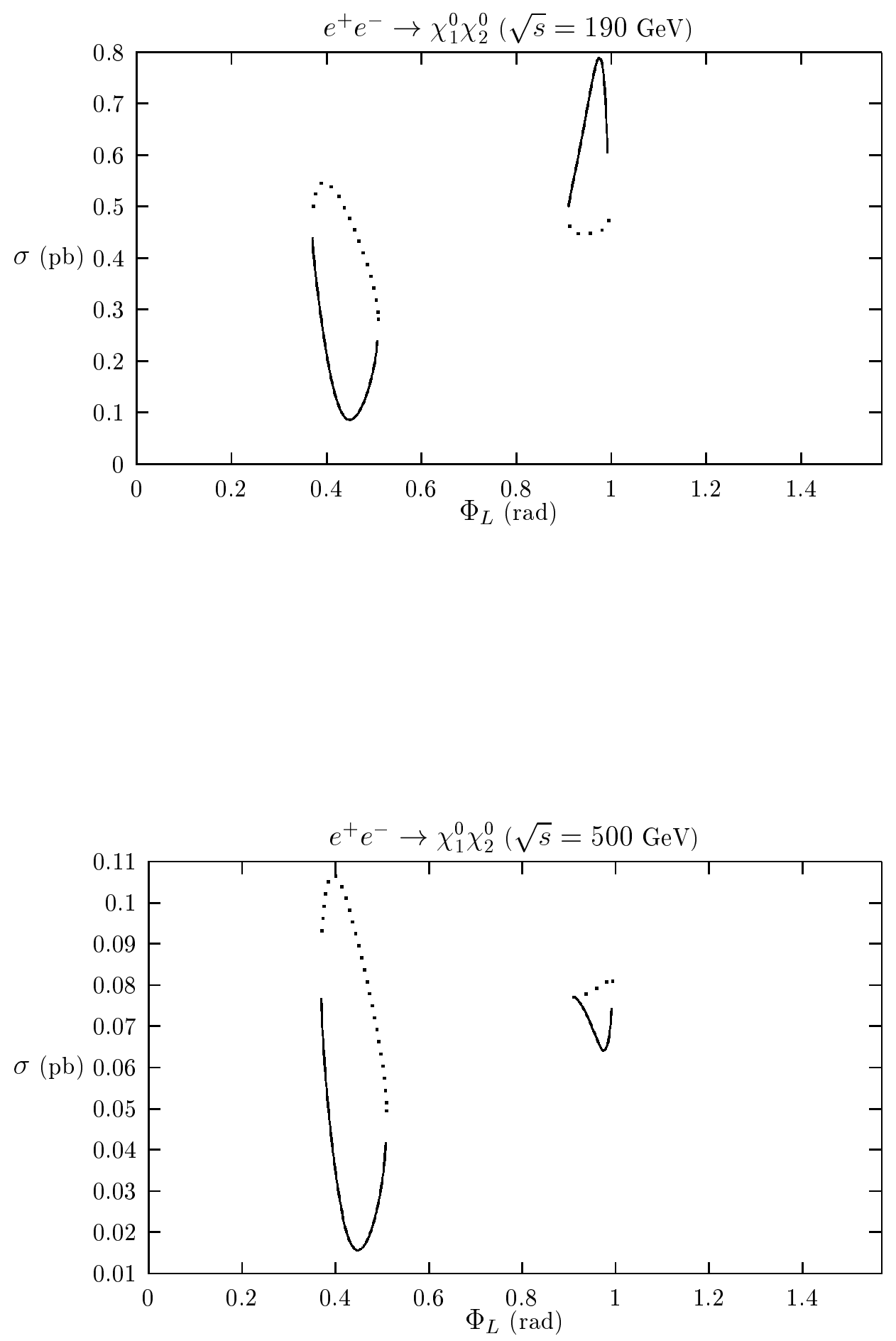

Figure 10: $\quad \chi_{1}^{0}, \chi_{2}^{0}$ production cross-section at $\sqrt{s}=190,500 \mathrm{GeV}$, versus the chargino mixing angle $\phi_{L}$, and for the same physical input as fig. 3. Plain (dotted) lines correspond to the first (second) $M_{1}$ solution of fig. 3 . 